\title{
Sail optimization for upwind sailing: application in a Tornado, the Olympic class catamaran
}

\author{
Ana Laverón-Simavilla • Victoria Lapuerta • \\ Sebastián Franchini · Angel Sanz
}

\begin{abstract}
A study of a boat's motion is carried out in order to analyze the aerodynamic properties of the optimal sail for obtaining the maximum velocity when sailing to windward. The mechanics study shows the optimal $C_{\mathrm{L}}$ and $C_{D}$ for a given sail and how the shape of the aerodynamic polar of the sail should be. A parametrical analysis of the aerodynamics of a sail is then carried out varying the maximum camber, position of the maximum camber in the chord direction and position of the maximum camber in the mast direction. The parametric analysis is done numerically with a vortex lattice method (VLM) and experimentally in a wind tunnel. The results show that the influence of the relevant parameters studied can be reduced to the variation of two parameters, $A$ and $B$, defining the polar of the sail, $C_{\mathrm{D}}=B+A^{2} C_{\mathrm{L}}{ }^{2}$; and the influence of parameters $A$ and $B$ on the maximum VMG obtainable are calculated.
\end{abstract}

Keywords Sailboats - Optimization - Aerodynamics

\section{List of symbols}

$A$ coefficient of the polar of the sail defined as $C_{\mathrm{D}}=B+A^{2} C_{\mathrm{L}}^{2}$

$b \quad$ sail span

$B$ coefficient of the polar of the sail defined as $C_{\mathrm{D}}=B+A^{2} C_{\mathrm{L}}^{2}$

$C$ profile chord

$C_{\mathrm{D}} \quad$ drag coefficient
$C_{\mathrm{Hx}}$ hydrodynamic force coefficient in the direction normal to the yacht speed

$C_{\mathrm{Hy}}$ hydrodynamic force coefficient in the direction of the yacht speed

$C_{\mathrm{L}} \quad$ lift coefficient

$C_{\text {Lopt }}$ optimal sailing lift coefficient

$C_{\mathrm{M}} \quad$ heeling moment coefficient made dimensionless with the apparent wind velocity

$C_{\mathrm{M}}^{*} \quad$ heeling moment coefficient made dimensionless with the true wind velocity

$C_{\mathrm{r}} \quad$ chord of the root section of the sail

$C_{\mathrm{t}} \quad$ chord of the tip section of the sail

D drag

$\vec{F}_{\mathrm{A}} \quad$ aerodynamic force

$\vec{F}_{\mathrm{H}} \quad$ hydrodynamic force

Fn Froud number, $F_{n}=\frac{\left|\vec{V}_{s}\right|}{\sqrt{g D_{\mathrm{h}}}}$, where $g$ is the gravity and $D_{\mathrm{h}}$ is the characteristic length scale of hydraulic depth

$K \quad \frac{\rho_{\mathrm{H}_{\mathrm{O}} \mathrm{O}} S_{\mathrm{H}}}{\rho_{\mathrm{g}} S_{\mathrm{A}}}$

L lift

$\mathrm{m}$ maximum camber of the profile expressed as a percentage of its chord length

$m_{\tau} \quad$ slope of the twist profile

$R n_{\mathrm{A}} \quad$ aerodynamic Reynolds number, $R_{n \mathrm{~A}}=\rho_{a} \frac{\left|V_{\mathrm{A}}\right| \bar{C}_{\mathrm{A}}}{\mu_{\mathrm{A}}}$, being $\bar{C}_{\mathrm{A}}$ the media chord of the sail and $\mu_{\mathrm{A}}$ the dynamic viscosity of the water

$R n_{\mathrm{H}} \quad$ hydrodynamic Reynolds number, $R_{n \mathrm{H}}=\rho_{\mathrm{H}_{2} \mathrm{O}}$ $\frac{V_{s} L_{\text {hull }}}{\mu_{\mathrm{H}, \mathrm{O}}}$, being $L_{\text {hull }}$ the length of the hull and $\mu_{\mathrm{H}_{2} \mathrm{O}}$ the dynamic viscosity of the air

$S_{\mathrm{A}} \quad$ characteristic area of the sail

$S_{\mathrm{H}} \quad$ characteristic area of the hull

$\overrightarrow{V_{\mathrm{A}}} \quad$ apparent wind speed

VMG Velocity Made Good

$V_{\mathrm{mg}} \quad$ velocity made good

$\overrightarrow{V_{\mathrm{S}}} \quad$ yacht speed 


\begin{tabular}{|c|c|}
\hline $\overrightarrow{V_{\mathrm{T}}}$ & wind speed \\
\hline$\rho_{\mathrm{a}}$ & air density \\
\hline$\rho_{\mathrm{H}_{2} \mathrm{O}}$ & water density \\
\hline$\alpha$ & angle of attack of the sail \\
\hline$\lambda$ & drift angle \\
\hline$\eta_{\mathrm{A}}$ & aerodynamic efficiency of the sail \\
\hline$\eta_{\mathrm{H}}$ & hydrodynamic efficiency of the hull \\
\hline$\eta_{\mathrm{m}}$ & $\begin{array}{l}\text { location of the profile with maximum camber along } \\
\text { the mast direction as a percentage of the sail span }\end{array}$ \\
\hline$\xi_{\mathrm{m}}$ & $\begin{array}{l}\text { location of the maximum camber along the chord } \\
\text { of the profile expressed as a percentage of the } \\
\text { chord }\end{array}$ \\
\hline$\mu$ & taper ratio \\
\hline$\tau$ & twist angle of each profile \\
\hline
\end{tabular}

\section{Introduction}

Several studies have been carried out on sail shapes; some of them use numerical aerodynamic codes in order to obtain the force distribution on the sail and the results are introduced in a structural analysis code to calculate the sail deformation, repeating the process until the equilibrium shape is reached $[1,2]$. The deformation of the sail-mast and its influence on the sail shape and on the aerodynamic forces has also been studied using the mentioned scheme. A different approach using the thin airfoil theory to study the aerodynamic loads, and the thin shell theory to study the sail deformation allows the development of analytic expressions [3]. Other numerical works have studied the aerodynamic forces of two interacting sails, the main sail and the jib [4]. A great effort has also been made in the experimental field; different experiments have been carried out, obtaining the aerodynamic forces of sails, studying their wake $[5,6]$ and developing twisted flow wind tunnels $[7,8]$.

Although there are many works dealing with the aerodynamics of sails, there is not a clear understanding of the influence of the aerodynamic forces on the performance of the yacht. Some attempts have been made in that direction [9]. An interesting study of the maximum thrust of sails when sailing close to wind, and the influence of the heeling force and moment on the maximum thrust is done using linearized expansions in Sparenberg et al. [6]. Another interesting study of the circulation distribution along the mast for maximum thrust, disregarding the heeling angle, is done in Wood et al. [10]. Later works can be found in which the thrust force is maximized in an attempt of finding a way of optimizing the sail design [11]. The direct application of the research done in the aerodynamics of airplane wings led to sails similar to the optimal wings, but those wings were designed to maximize the lift-drag ratio because in that way the cost was minimized. The works using the criterion of thrust maximization for a given heeling moment are only assuming that this is the true optimization criterion. Maximizing the thrust is a simple way of decoupling the aerodynamic problem from the hydrodynamic problem; however, the truth is that these two problems cannot be decoupled if the windward performance of a sailing craft is to be optimized. Maximizing the thrust is not the criterion for traveling as fast as possible without capsizing, as will be demonstrated in this work.

The performance of the yacht is a complex problem and a first approach to study the bi-dimensional movement of the boat is carried out in this work, assuming the heel angle to be small. There are cases in which the heel may be considered small enough to be of little importance; especially where the majority of the righting moment comes from crew weight, as in sailing dinghies and small catamarans. As the heeling angle increases the results here obtained are less applicable. In such a way some analytical expressions can be obtained for the yacht performances. These expressions constitute powerful tools which avoid the need of Velocity Prediction Program (VPP) programs for design purposes, providing the sail designer with direct optimization criteria; decoupling the hydrodynamic/aerodynamic code from the optimization tool. In this work, combining the yacht's performance equations with the aerodynamic analysis of the sail, the optimum sailing configuration is obtained for a given sail maximizing the Velocity Made Good (VMG) of the yacht. A numerical parametrical study of the aerodynamics of a sail is also carried out in this work, and the influence of the studied parameters on the maximum obtainable VMG for a given yacht is also obtained. Finally, the same parametrical study carried out numerically has been carried out experimentally in a wind tunnel for the same sail. The three-dimensional study of the yacht performance is actually being done and the results will soon be obtained.

\section{The motion of the boat}

The motion of a yacht sailing upwind is studied here in a plane parallel to the water surface, while assuming the heel angle to be small. The kinematics are first studied relating the yacht speed $\overrightarrow{V_{\mathrm{S}}}$, the wind speed, $\overrightarrow{V_{\mathrm{T}}}$, the apparent wind speed, $\overrightarrow{V_{\mathrm{A}}}$, and the VMG, $V_{\mathrm{mg}}$, with the later velocity, which is the one which will be maximized in the present work. The equilibrium of forces and moments are established along with the optimizing condition, and the optimal configuration, for a given hull and sail, is finally obtained.

In a fixed inertial system of reference the three mentioned velocities are related as $\overrightarrow{V_{\mathrm{T}}}=\overrightarrow{V_{\mathrm{S}}}+\overrightarrow{V_{\mathrm{A}}}$ as 
represented in Fig. 1 along with the angles involved in the geometry,

The variable to be optimized is the VMG, which can be written as $V_{\mathrm{mg}}=V_{\mathrm{S}} \cos \%$. Another important geometrical relation is

$\frac{V_{\mathrm{mg}}}{V_{\mathrm{T}}}=\frac{\cos \gamma \sin (\gamma-\beta)}{\sin \beta}$.

The following assumptions are made: the yacht is rigid, its center of mass does not change, the yacht moves with constant velocity, and the water surface is a perfect plane. The forces and moments acting on the yacht are sketched in Fig. 2. The aerodynamic force, $\vec{F}_{\mathrm{A}}$, is the force exerted by the air on the sail, hull and all the appendages. $\vec{F}_{\mathrm{H}}$ is the hydrodynamic force on the hull. All forces and moments are expressed in a reference system with origin in the center of mass of the system, the $x y$ plane is parallel to the water plane with the $y$ axis parallel to the true velocity of the yacht and pointing in its same direction, the $z$ axis is perpendicular to the $x y$ plane.

For uniform movement the equilibrium of forces and moments give the following equations

$\vec{F}_{\mathrm{A}}-\vec{F}_{\mathrm{H}}=0$

$\sum M_{\mathrm{z}}=0$.

The hydrodynamic forces of the rudder have been neglected in Eq. 2 compared to the hydrodynamic forces of the hull, even though those forces need to be retained in Eq. 3 to keep drift balance. Equation 3 has been written for completeness, but it will not be used in the following as it would provide the leeway angle determination; which would only be necessary to solve the hydrodynamic

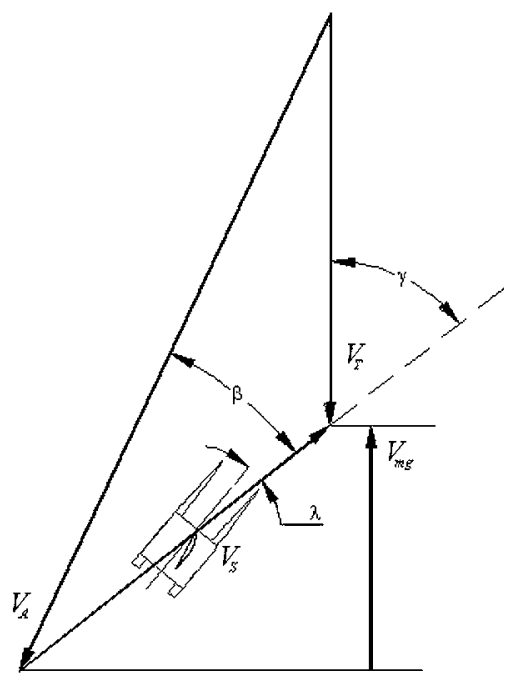

Fig. 1 Triangle of velocities and relevant angles of the geometry problem. As it will be demonstrated the hydrodynamic problem is decoupled from the aerodynamic problem, and it should be solved independently by other means.

The aerodynamic non-dimensional force coefficients, $C_{\mathrm{L}}$ and $C_{\mathrm{D}}$, can be expressed as

$$
\begin{aligned}
C_{\mathrm{L}} & =\frac{\mathrm{L}}{\frac{1}{2} \rho_{\mathrm{a}} V_{\mathrm{A}}^{2} S_{\mathrm{A}}}, \\
C_{\mathrm{D}} & =\frac{\mathrm{D}}{\frac{1}{2} \rho_{\mathrm{a}} V_{\mathrm{A}}^{2} S_{\mathrm{A}}} .
\end{aligned}
$$

In the above equation, $L$ is the lift force and $D$ the drag force. Both coefficients depend on the aerodynamic Reynolds number, $R n_{\mathrm{A}}$, and on the angle of attack of the sail, $\alpha$, which is the angle between the apparent wind vector and the zero lift line of the sail. In the same manner the hydrodynamic forces can be written as

$F_{\mathrm{Hx}}=\frac{1}{2} \rho_{\mathrm{H}_{2} \mathrm{O}} V_{\mathrm{s}}^{2} S_{\mathrm{H}} C_{\mathrm{Hx}}$
$F_{\mathrm{Hy}}=\frac{1}{2} \rho_{\mathrm{H}_{2} \mathrm{O}} V_{\mathrm{s}}^{2} S_{\mathrm{H}} C_{\mathrm{Hy}}$

with $C_{\mathrm{Hx}}$ and $C_{\mathrm{Hy}}$ being the non-dimensional force coefficients which depend on the drift angle, $\lambda$, the hydrodynamic Reynolds number, $R n_{\mathrm{H}}$, the hull shape and the Froud number, Fn. Defining the hydrodynamic efficiency of the hull as the ratio between the lateral and the longitudinal hydro-dynamical force coefficients of the hull, $\eta_{\mathrm{H}}=\frac{C_{\mathrm{Hx}}}{C_{\mathrm{Hy}}}$, and defining the aerodynamic efficiency of the sail as the ratio between the lift coefficient, $C_{\mathrm{L}}$, and the drag coefficient, $C_{\mathrm{D}}$, of the sail, $\eta_{\mathrm{A}}=\frac{C_{\mathrm{L}}}{C_{\mathrm{D}}}$, Eqs. 2-5 lead to $\beta=\arctan \frac{1}{\eta_{\mathrm{H}}}+\arctan \frac{1}{\eta_{\mathrm{A}}}$

It is necessary to note that the aerodynamic forces are exerted by the whole yacht, but the lift can be approximated as if it was only exerted by the sail. The drag coefficient should be computed as the one produced by the sail and by the appendages.

The second condition for the equilibrium of forces can be obtained equating the modulus of the forces

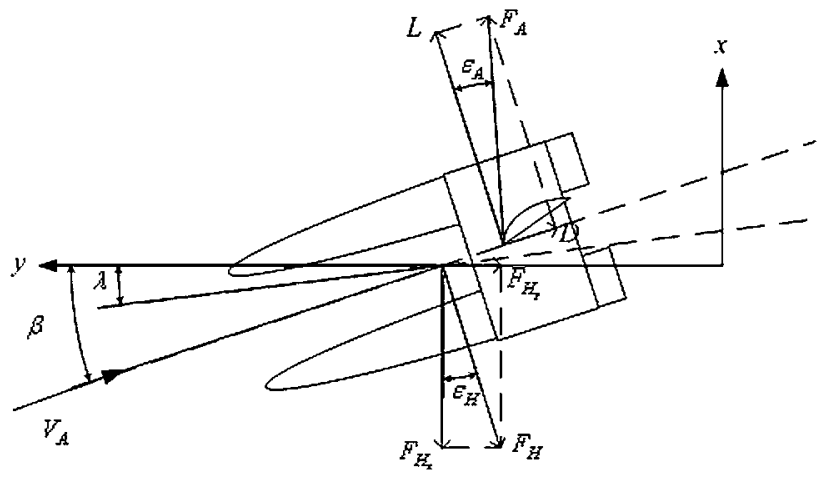

Fig. 2 Forces acting on the water plane 


$$
\left(\frac{V_{\mathrm{A}}}{V_{\mathrm{S}}}\right)^{2} \sqrt{\frac{\eta_{\mathrm{A}}^{2}+1}{\eta_{\mathrm{H}}^{2}+1}}=K \frac{C_{\mathrm{Hy}}}{C_{\mathrm{D}}}
$$

where $K=\frac{\rho_{\mathrm{H}_{2}} S_{\mathrm{H}}}{\rho_{\mathrm{A}} S_{\mathrm{A}}}$. Finally, using the relation $\frac{V_{\mathrm{A}}}{V_{\mathrm{S}}}=\frac{\sin \gamma}{\sin (\gamma-\beta)}$

$\cot \gamma= \pm \sqrt[4]{\frac{\eta_{\mathrm{A}}^{2}+1}{\eta_{\mathrm{H}}^{2}+1}} \sqrt{\frac{C_{\mathrm{D}}}{C_{\mathrm{Hy}}}} \frac{1}{\sqrt{K} \sin \beta}+\cot \beta$

Recalling that the angles $\beta$ and $\gamma$ take values in the intervals [9]:

$20^{\circ}<\beta<40^{\circ}$

$35^{\circ}<\gamma<55^{\circ}$,

One can verify that only the negative sign has physical significance in Eq. 8. Combining Eqs. 1, 6 and 8, and for a given yacht, one can obtain the following relation:

$$
\begin{aligned}
\frac{V_{\mathrm{mg}}}{V_{\mathrm{T}}} & =f\left(C_{\mathrm{L}}, C_{\mathrm{D}}, C_{\mathrm{Hx}}, C_{\mathrm{Hy}}\right) \\
& =\frac{\cot ^{2} \gamma}{1+\cot ^{2} \gamma}\left(\frac{1}{\tan \beta \cot \gamma}-1\right),
\end{aligned}
$$

with

$$
\begin{aligned}
& \tan \beta=\frac{C_{\mathrm{Hx}} C_{\mathrm{D}}+C_{\mathrm{L}} C_{\mathrm{Hy}}}{C_{\mathrm{Hx}} C_{\mathrm{L}}-C_{\mathrm{Hy}} C_{\mathrm{D}}}, \\
& \cot \gamma= \pm \frac{1}{\tan \beta}\left[\sqrt[4]{\frac{C_{\mathrm{L}}^{2}+C_{\mathrm{D}}^{2}}{C_{\mathrm{Hx}}^{2}+C_{\mathrm{Hy}}^{2}}} \sqrt{\frac{1+\tan ^{2} \beta}{K}}+1\right] .
\end{aligned}
$$

As the aim of the present work is to study the influence of different parameters defining the sail geometry, $C_{\mathrm{Hx}}$ and $C_{\mathrm{Hy}}$ will be assumed as given parameters of the configuration. The relation between $C_{\mathrm{L}}$ and $C_{\mathrm{D}}$ is plotted in Fig. 3 for $C_{\mathrm{Hx}}=0.1, K=100$ and $\eta_{\mathrm{H}}=3$ for different values of the non-dimensional VMG. The figure shows that

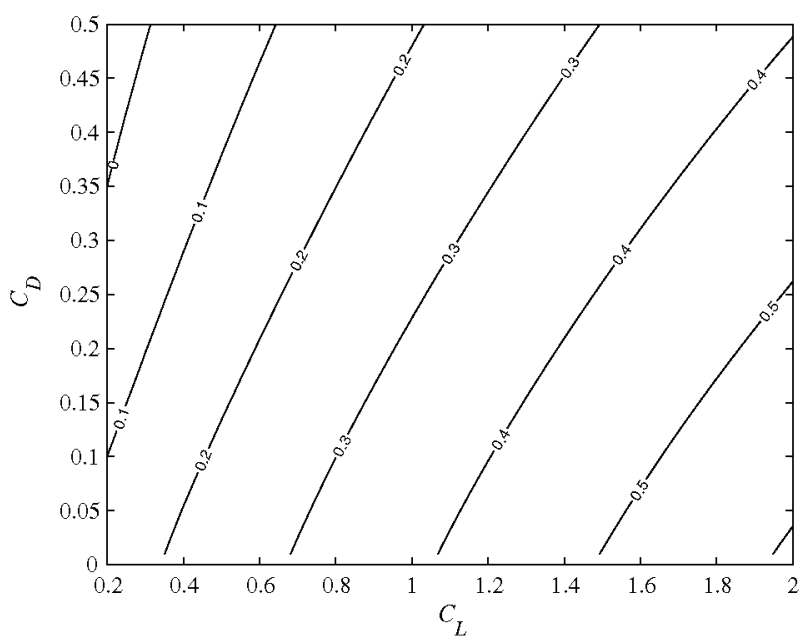

Fig. 3 Coefficient of the aerodynamic drag versus the coefficient of aerodynamic lift for different values of the non-dimensional VMG increasing the lift coefficient and the drag coefficient of the sail may lead to decreasing values of the dimensionless VMG, and it also shows that decreasing the values of the lift coefficient does not have to mean decreasing the dimensionless VMG, if the drag coefficient is also decreased. It can be demonstrated that there are no extremes in Eq. 9.

The influence of the hydrodynamic force coefficients on the shape of the constant dimensionless VMG curves can be seen in Fig. 4 where the curves for $K=100, \eta_{\mathrm{H}}=3$ and for two different values of the hydrodynamic coefficient $C_{\mathrm{Hx}}$ are represented.

Changing from a value of the hydrodynamic coefficient, $C_{\mathrm{Hx} 1}$, to a different one, $C_{\mathrm{Hx} 2}$, with constant the hydrodynamic efficiency, $\eta_{\mathrm{H}}$, the curves corresponding to a constant value of VMG plotted in the $C_{\mathrm{L}}-C_{\mathrm{D}}$ plane are homothetic with ratio $r=C_{\mathrm{Hx} 2} / C_{\mathrm{Hx} 1}$. It can be seen in Eq. 8 that with $C_{\mathrm{Hy} 2}=r C_{\mathrm{Hy} 1}, \quad C_{\mathrm{D} 2}=r C_{\mathrm{D} 1} \quad$ and $C_{\mathrm{L} 2}=r C_{\mathrm{L} 1}$, VMG remains constant.

The figure shows how the curves obtained for a fixed value of the dimensionless VMG move towards bigger values of $C_{\mathrm{L}}$ or/and smaller values of $C_{\mathrm{D}}$ as the hydrodynamic coefficients of forces increase their values, with the value of the hydrodynamic efficiency held constant. This means that the sail of the yacht will have to be capable of providing bigger values of the lift coefficient or/and smaller values of the drag coefficient in order to achieve the same values of the dimensionless VMG.

The influence of the hydrodynamic efficiency of the hull is plotted in Fig. 5, the curves have been obtained for $K=100, C_{\mathrm{Hx}}=0.1$ and two hydrodynamic efficiencies $\eta_{\mathrm{H}}=3$ with solid line, and $\eta_{\mathbf{H}}=4$ with dotted line. The

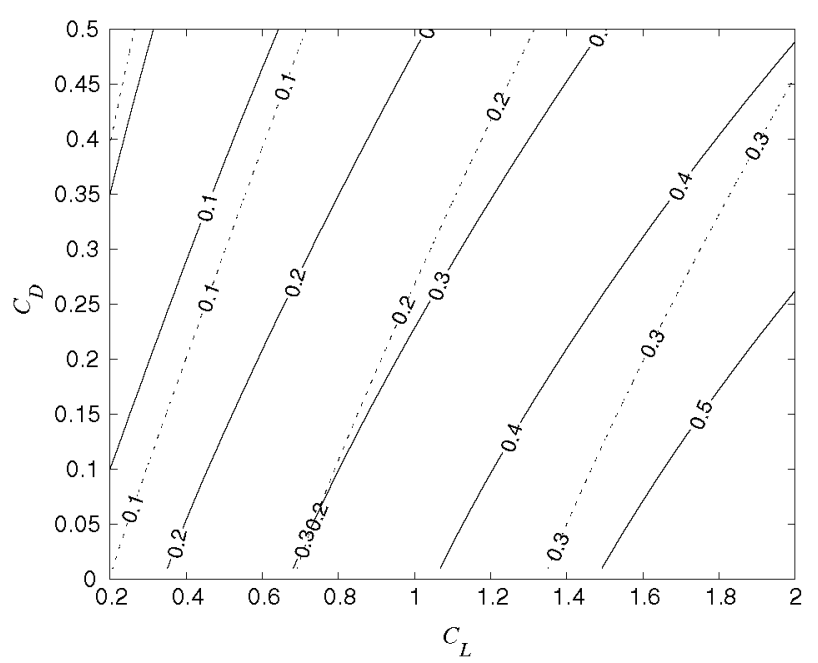

Fig. 4 Coefficient of the aerodynamic drag versus the coefficient of aerodynamic lift for different values of the non-dimensional VMG, for two different values of the lateral hydrodynamic force coefficient $\left(C_{\mathrm{Hx}}=0.1\right.$ solid line, $C_{\mathrm{Hx}}=0.2$ dotted line $), K=100$ and $\eta_{\mathrm{H}}=3$ 


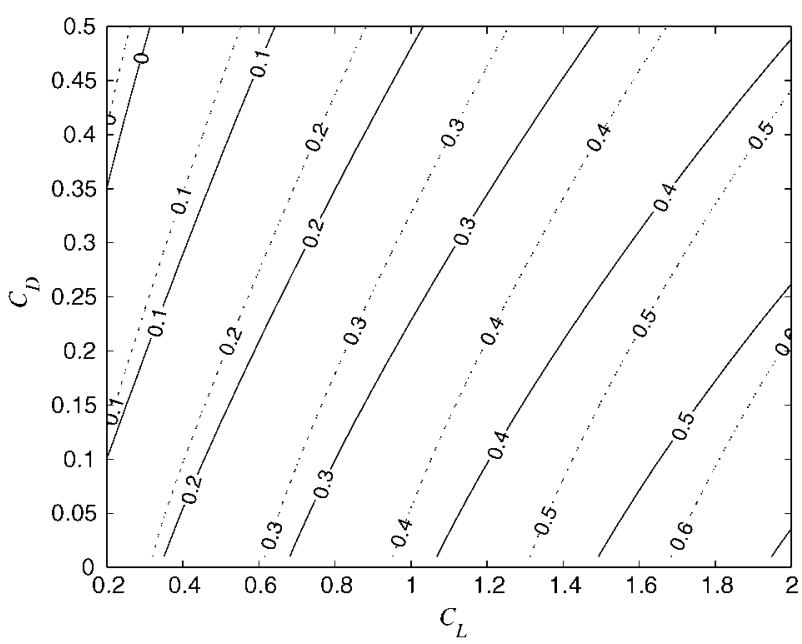

Fig. 5 Coefficient of the aerodynamic drag versus the coefficient of aerodynamic lift for different values of the non-dimensional VMG, for two different values of the hydrodynamic efficiency $\left(\eta_{\mathrm{H}}=4\right.$ dotted line, $\eta_{\mathrm{H}}=3$ solid line), $C_{H x}=0.1$ and $K=100$

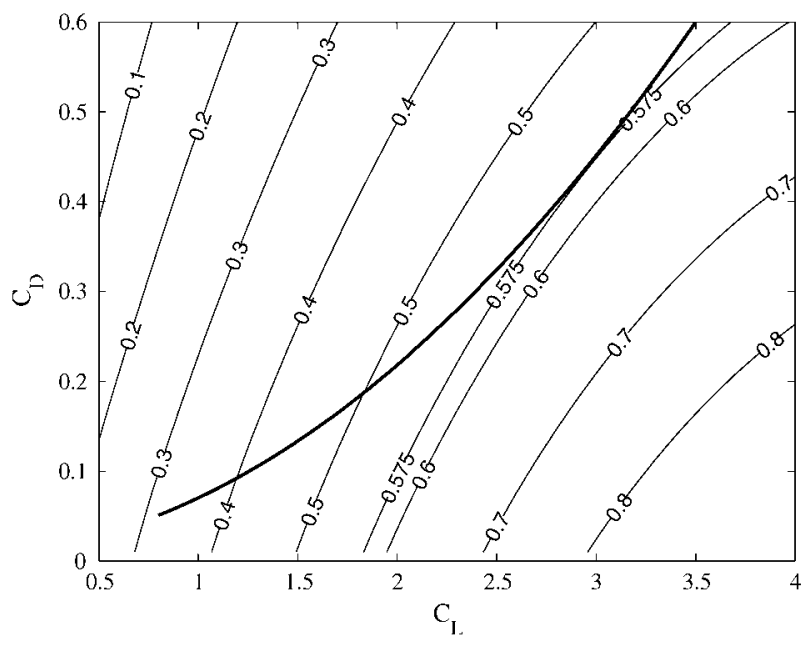

Fig. 6 Determination of the optimum sailing condition for a given sail and a given hull. In this case the maximum dimensionless VMG is 0.575

effect of decreasing the hydrodynamic efficiency is similar to the effect of increasing the hydrodynamic force coefficients while holding the hydrodynamic efficiency constant.

For a given geometry of the sail the aerodynamic coefficients of lift and drag are not independent, but are related to the so-called sail polar, $C_{\mathrm{D}}=f\left(C_{\mathrm{L}}\right)$. In order to obtain the sailing condition, both equations must be satisfied: the polar of the sail and the equation obtained from the yacht's mechanics; therefore, their intersection points will give the sailing condition of the yacht. If the VMG is to be maximized for a given value of the true wind, the optimal sailing point, $C_{\mathrm{Lopt}}$, should be the one determined by the tangency of the polar of the sail and the curves

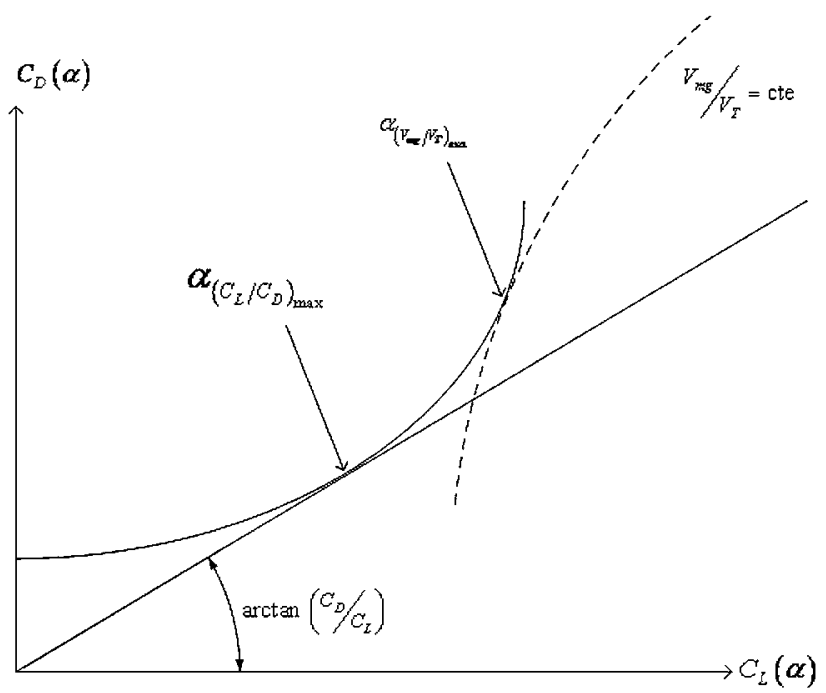

Fig. 7 Draft showing the different results obtained for the navigation point maximizing the aerodynamic efficiency of the sail and maximizing the VMG

$C_{\mathrm{D}}=f\left(C_{\mathrm{L}} ; V_{\mathrm{mg}} / V_{\mathrm{T}}\right)$ as represented in Fig. 6. The maximum dimensionless VMG in this case is 0.575. For increasing values of the sail lift beyond the optimal sailing point the dimensionless VMG decreases. The same occurs for decreasing values of the sail lift under the optimal sailing point. The difference in the optimal navigation point obtained for a given sail maximizing $V_{\mathrm{mg}} / V_{\mathrm{T}}$, from the one obtained maximizing the aerodynamic efficiency of the sail is represented in Fig. 7. The figure clearly shows how the extended criterion of maximizing the aerodynamic efficiency of the sail (trying to obtain the greatest lift coefficient and the smallest drag coefficient) does not guarantee the maximum VMG.

For a given sail geometry, there is a maximum value for the VMG if it can be assured that the yacht can withstand the heeling moment, and that maximum value can be calculated by approximating the polar of the sail with the parabola $C_{\mathrm{D}}=B+A^{2} C_{\mathrm{L}}^{2}$, as stated by the lifting line theory. The equation for the optimal sailing condition is given by

$$
\begin{aligned}
& \left(\tan \beta-2 \tan \gamma-\tan ^{2} \gamma \tan \beta\right)\left(C_{\mathrm{D}}-2 B\right) \\
& \quad+\left(1+2 \tan \beta \tan \gamma-\tan ^{2} \gamma\right) \eta_{A} C_{D}\left(\frac{1}{2}+A^{2} C_{\mathrm{D}}\right) \\
& \quad=0
\end{aligned}
$$

and

$\eta_{\mathrm{A}}=\frac{\sqrt{C_{\mathrm{D}}-B}}{A C_{\mathrm{D}}}$

Equation 12 has been obtained by deriving Eq. 9 with respect to $C_{\mathrm{D}}$ and setting the derivative to zero. In order to obtain the derivative $\frac{\partial V_{\mathrm{mg}} / V_{\mathrm{r}}}{\partial C_{\mathrm{D}}}$ Eqs. 10, 11 and their derivatives, $\frac{\partial \tan \beta}{\partial C_{\mathrm{D}}}$ and $\frac{\partial \cot \gamma}{\partial C_{\mathrm{D}}}$, have been used, together with 
the polar expression, $C_{\mathrm{D}}=B+A^{2} C_{\mathrm{L}}^{2}$, and its derivative $d C_{\mathrm{L}} / d C_{\mathrm{D}}=1 /\left(2 A^{2} \eta_{\mathrm{A}} C_{\mathrm{D}}\right)$.

Equation 13 has been obtained substituting $C_{\mathrm{L}}=\eta_{\mathrm{A}} C_{\mathrm{D}}$ in the polar expression, $C_{\mathrm{D}}=B+A^{2} C_{\mathrm{L}}^{2}$.

From Eqs. 12 and 13 the value of the drag coefficient for the optimal sailing point can be calculated, and introducing this value in the polar of the sail, the lift coefficient and the aerodynamic efficiency of the sail can also be calculated. The best possible dimensionless VMG and aerodynamic coefficients are plotted in Fig. 8 as a function of $B$ $(A=0.225)$, and in Fig. 9 they are represented as a function of $A$ for two different values of $B(B=0.016$ and $B=0.15$ ), with the values for the hydrodynamic coefficients $C_{\mathrm{Hx}}=0.1, \eta_{\mathrm{H}}=3$, and $K=100$. The numerical values used to obtain the values represented in Fig. 8 correspond to the ones obtained for the case studied below in Sect. 3.2.1; in that case the maximum camber position is varied along the mast direction yielding to changes in the aerodynamic polar of the sail in such a way that the parameter $A$ remains constant. The numerical value, $B=0.016$, used in Fig. 9 corresponds to the one obtained for the case studied in Sect. 3.2.2; in that case varying the value of maximum camber of the sail changes the aerodynamic polar of the sail in such a way that the parameter $B$ remains constant. The value $B=0.016$ would approximate the real one if the flow was attached to the sail, but the experimental results showed that there are big areas of the sail with detached flow. From the experimental results the non-potential drag coefficient can be calculated using $B=0.15$. The result for this value has also been included in Fig. 9. The curves obtained for the optimal sailing point are plotted with solid $(B=0.016)$ and dashed $(B=0.15)$ lines. The curve obtained using the maximum aerodynamic efficiency criteria, criteria that has been used when applying directly the airplane wings aerodynamics research is plotted with a dotted line.

It can be seen from the figures how the optimal sailing point is always obtained for higher values of the aerodynamic force coefficients than the ones corresponding to the maximum aerodynamic efficiency criteria. It is also interesting to point out that the difference in the VMG obtained with both criteria decrease as the polar parameters, $A$ and $B$, increase. The difference obtained with both criteria can be easily seen in Eq. 12 as the maximum aerodynamic efficiency criteria can be written as $C_{\mathrm{D}}=2 B$.

In order to increase the dimensionless VMG, it is convenient to increase the lift coefficient and this effect can be obtained increasing the angle of attack of the sail but increasing the angle of attack of the sail also increases the sail's heeling moment. For this reason there is a maximum value of the possible lift coefficient for a given sail configuration, $C_{\mathrm{Lmax}}$, which corresponds to the maximum heeling moment the yacht can withstand. If $C_{\mathrm{Lmax}}>C_{\mathrm{Lopt}}$ then the optimal sailing point defined by the tangency condition described above is feasible, but if $C_{\mathrm{Lmax}}<C_{\mathrm{Lopt}}$ the hull cannot withstand the heeling moment caused by
Fig. 8 VMG and aerodynamic coefficients of the sail for the optimal sailing point versus one of the parameters of the polar of the sail, $B$. The values are obtained for typical values of the hydrodynamic coefficients $\left(C_{\mathrm{Hx}}=0.1, \eta_{\mathrm{H}}=3, K=100\right)$, and for the other parameter defining the polar of the sail $(A=0.225)$. The dotted line corresponds to the maximum aerodynamic efficiency criteria
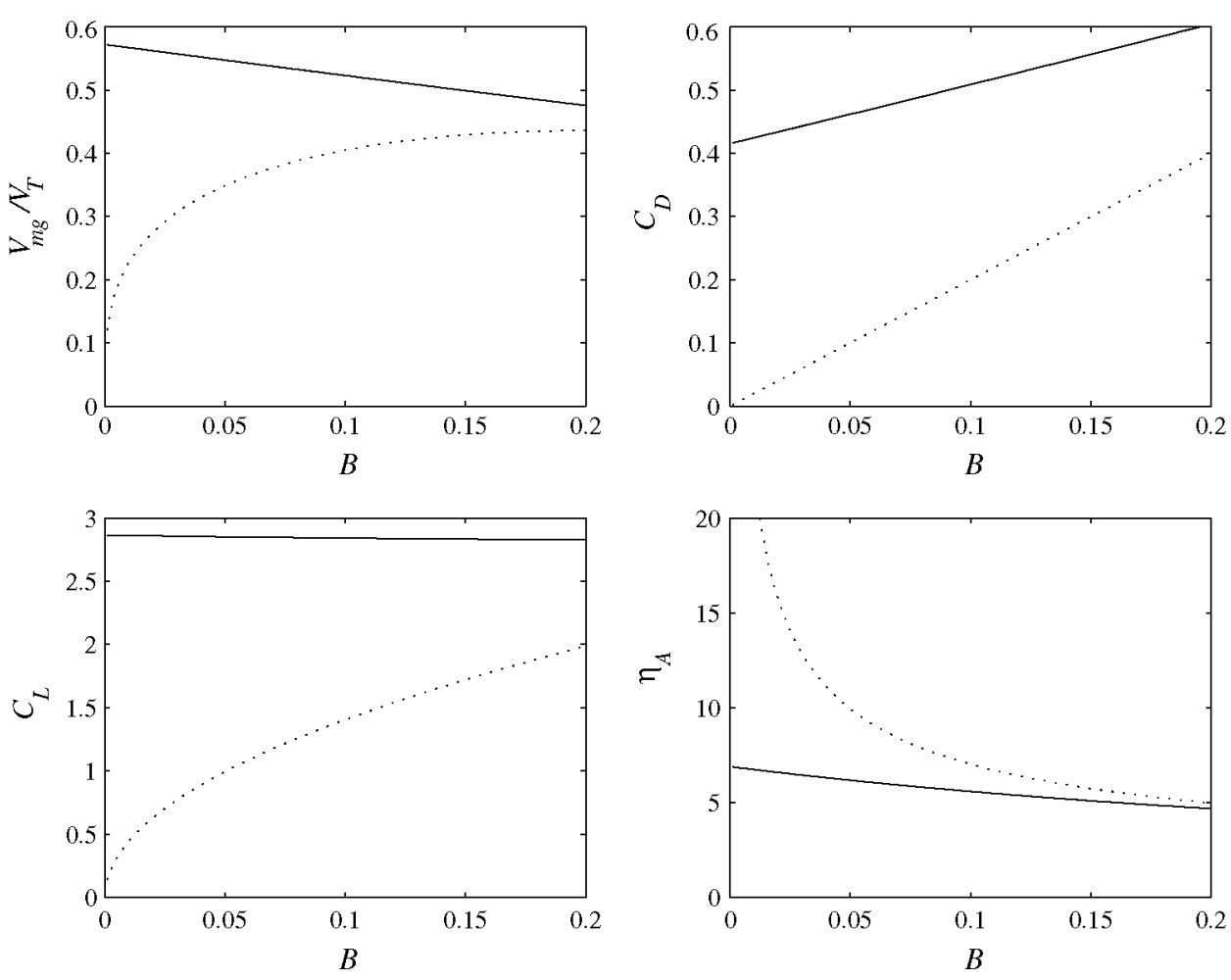
Fig. 9 VMG and aerodynamic coefficients of the sail for the optimal sailing point versus one of the parameters of the polar of the sail, $A$. The values are obtained for typical values of the hydrodynamic coefficients $\left(C_{\mathrm{Hx}}=0.1, \eta_{\mathrm{H}}=3, K=100\right)$, and for the other parameter defining the polar of the sail $(B=0.016$ with a solid line and $B=0.15$ with a dashed line). The dotted line corresponds to the maximum aerodynamic efficiency criteria
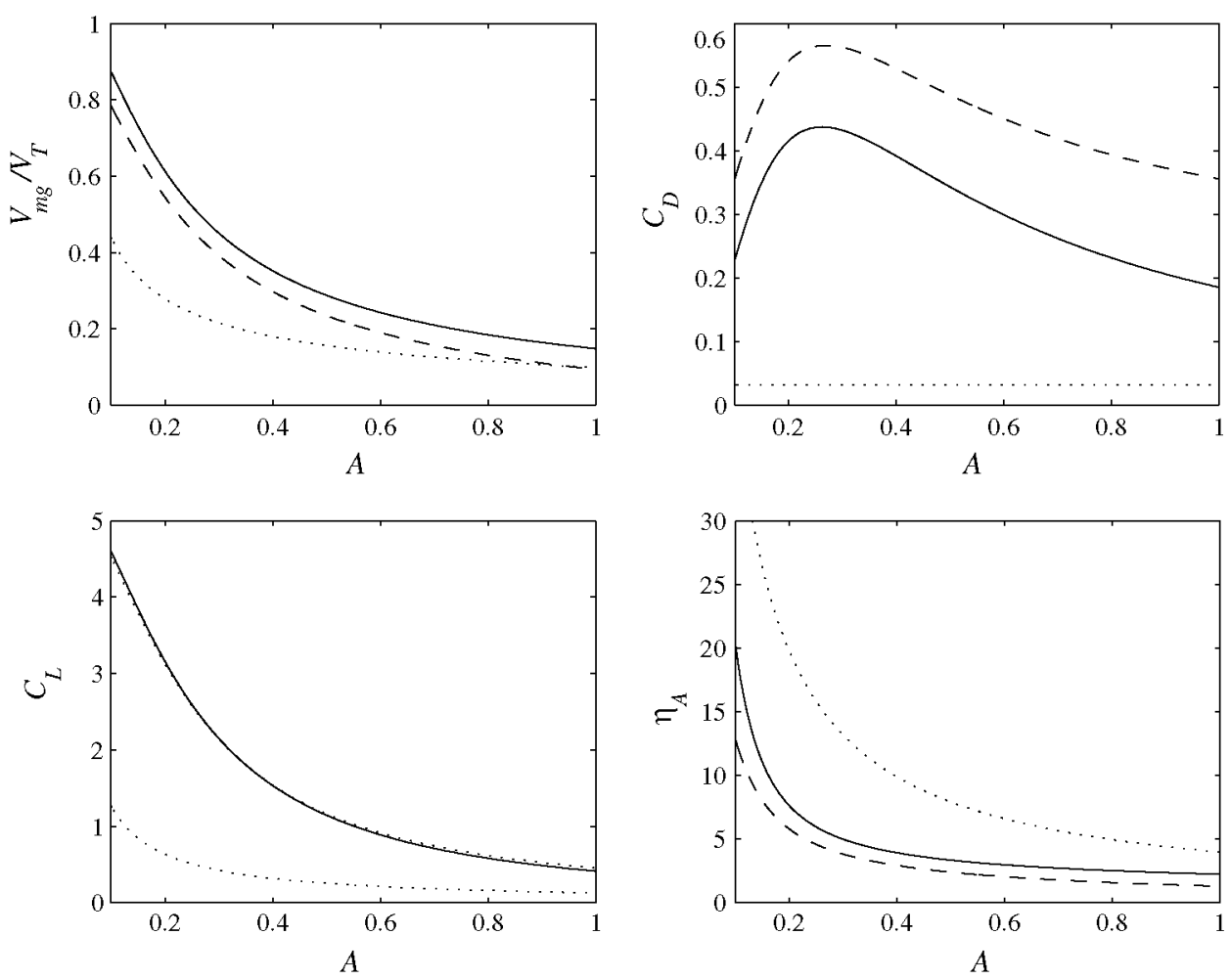

the sail and the optimal sailing point will be the one corresponding to $C_{\mathrm{Lmax}}$. The aerodynamic study of the sail provides its heeling moment coefficient, $C_{\mathrm{M}}$, which is the heeling moment made dimensionless using the apparent wind velocity

$M=\frac{1}{2} \rho_{\mathrm{a}} V_{\mathrm{A}}^{2} S_{\mathrm{A}} b C_{\mathrm{M}}$

where $b$ is the sail span. This is not a very useful parameter for the yacht configuration because the apparent wind velocity depends on the true wind and on the yacht's orientation; therefore a new heeling moment coefficient will be used, $C_{\mathrm{M}}^{*}$, which is the heeling moment coefficient made dimensionless with the true wind velocity

$C_{\mathrm{M}}^{*}=\frac{M}{\frac{1}{2} \rho_{\mathrm{a}} V_{\mathrm{T}}^{2} S_{\mathrm{A}} b}$.

The quotient $C_{\mathrm{M}}^{*} / C_{\mathrm{M}}$ is calculated from

$\frac{C_{\mathrm{M}}^{*}}{C_{\mathrm{M}}}=\left(\frac{V_{\mathrm{T}}}{V_{\mathrm{A}}}\right)^{2}=\left(\frac{\sin \beta}{\sin \gamma}\right)^{2}$,

and using Eqs. 6 and 8 one can write it as a function of $C_{\mathrm{L}}$, $C_{\mathrm{D}}, C_{\mathrm{Hy}}, \eta_{\mathrm{H}}$, and $K$. The ratio between both moment coefficients is represented in Fig. 10, which has been calculated for the following values of the parameters: $C_{\mathrm{Hx}}=0.1, \eta_{\mathrm{H}}=3$ and $K=100$.

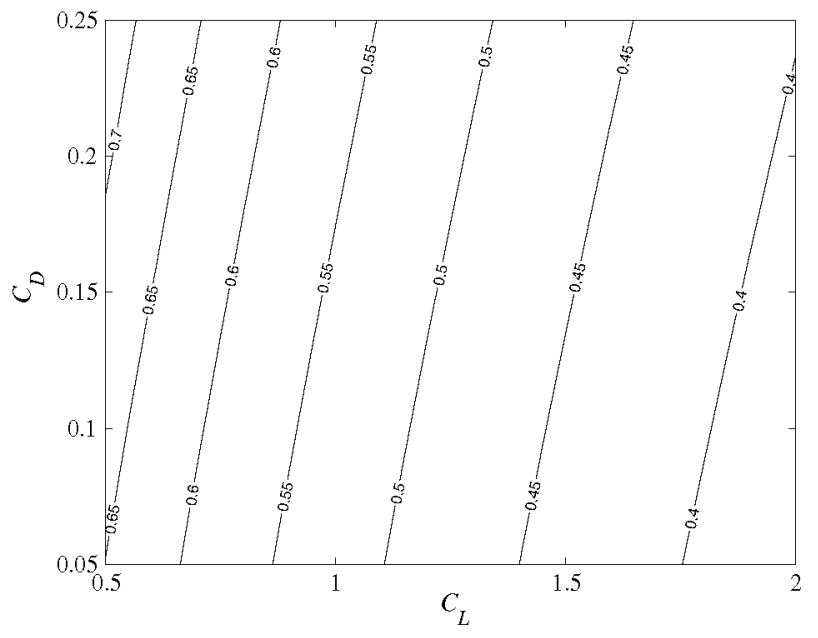

Fig. 10 Ratio between the heeling moment coefficients, $C_{\mathrm{M}}^{*} / C_{\mathrm{M}}$

Assuming, from the lifting line theory, that the heeling moment coefficient, $C_{M}$, has a linear variation with the lift coefficient, one can write it as

$C_{\mathrm{M}}=C_{\mathrm{M} 0}(A, B)+C_{\mathrm{M} 1}(A, B) C_{\mathrm{L}}$.

In order to extend the analysis done above using parameters $A$ and $B$, the dependence of $C_{\mathrm{M} 0}$ and $C_{\mathrm{M} 1}$ on parameters $A$ and $B$ has to be known. This analysis is done using the numerical results from Sects. 3.2.1 and 3.2.2. 
Fig. 11 Moment coefficient versus the parameter $B$, which represents the variation of the maximum camber position along the mast direction for $A=0.225$. The hydrodynamic coefficient values are $\eta_{\mathrm{H}}=3$, $C_{\mathrm{Hx}}=0.1$ and $K=100$. The dotted line represents the maximum moment coefficient the yacht can withstand
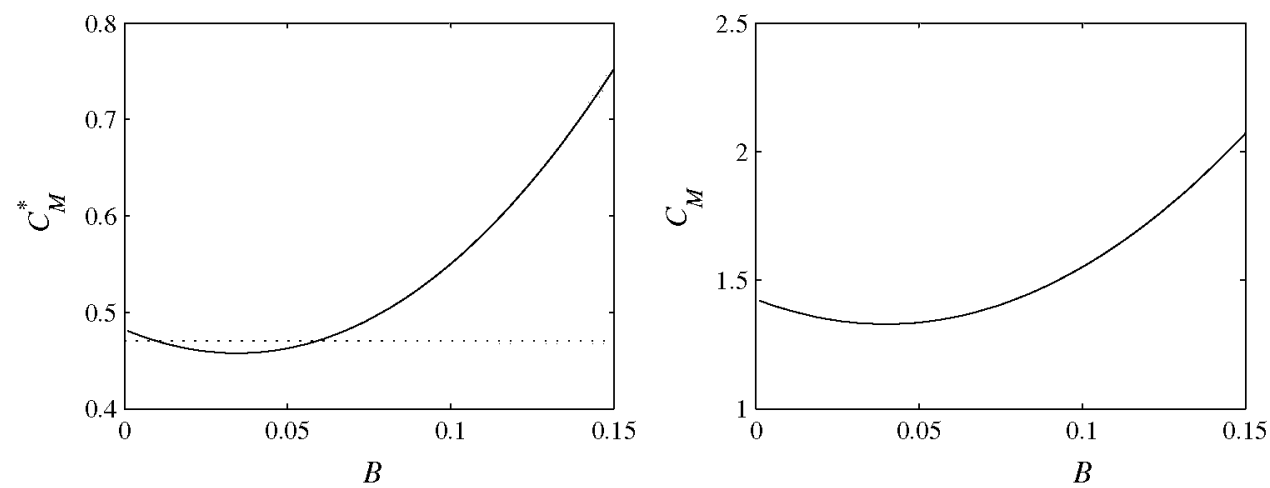

The results obtained in Sect. 3.2.1 show that coefficients $C_{\mathrm{M} 0}$ and $C_{\mathrm{M} 1}$ only depend on $B$; therefore the moment coefficients can be represented as a function of that parameter as shown in Fig. 11. A maximum value for $C_{\mathrm{M}}^{*}$ has been represented in this figure with a dashed line so it can be appreciated that even though decreasing $B$ is always desirable in terms of VMG, there is a limiting value of this parameter, which can be obtained by the intersection of both curves.

In a similar way, the results obtained in Sect. 3.2.2 show that coefficients $C_{\mathrm{M} 0}$ and $C_{\mathrm{M} 1}$ only depend on $A$; therefore, the moment coefficients can be represented as a function of that parameter as shown in Fig. 12. A maximum value for $C_{\mathrm{M}}^{*}$ has been represented in that figure with a dashed line, so it can be appreciated that in this case decreasing $A$ is always desirable in terms of VMG, and there is no limiting value for this parameter determined by the maximum heeling moment the hull of the yacht can withstand.

In order to study the influence of the hydrodynamic coefficients, the curves for the optimal sailing conditions obtained by varying the position of the maximum camber position along the mast direction represented in Fig. 8 are plotted for different values of the hydrodynamic force coefficient, $C_{\mathrm{Hx}}$, in Fig. 13; and are also plotted for different values of the hydrodynamic efficiency coefficient, $\eta_{\mathrm{H}}$, in Fig. 14. The influence of the hydrodynamic coefficients, $C_{\mathrm{Hx}}$ and $\eta_{\mathrm{H}}$, on the moment coefficients, $C_{\mathrm{M}}$ and
$C_{\mathrm{M}}^{*}$, are also plotted in Figs. 15 and 16 , respectively. In conclusion it can be stated that by decreasing the hydrodynamic force coefficients, $C_{\mathrm{Hx}}$ and $C_{\mathrm{Hy}}$, for constant values of the hydrodynamic efficiency coefficient, the dimensionless VMG increases and the moment coefficient, $C_{\mathrm{M}}^{*}$, decreases; both effects being beneficial for the design. On the other hand, for constant values of the force coefficient, $C_{\mathrm{Hx}}$, increasing the hydrodynamic efficiency coefficient, $\eta_{\mathrm{H}}$, increases the dimensionless VMG and also decreases the moment coefficient, $C_{\mathrm{M}}^{*}$; in this particular case, with constant $C_{\mathrm{Hx}}$, the increase in the hydrodynamic efficiency coefficient can only be done by decreasing the hydrodynamic force coefficient, $C_{\mathrm{Hy}}$.

In the same way, and in order to study the influence of the hydrodynamic coefficients, the curves for the optimal sailing conditions obtained by varying the value of the maximum camber of the sail represented in Fig. 9 are plotted for different values of the hydrodynamic force coefficient, $C_{\mathrm{Hx}}$, in Fig. 17; and are also plotted for different values of the hydrodynamic efficiency coefficient, $\eta_{\mathrm{H}}$, in Fig. 18. The influence of the hydrodynamic coefficients, $C_{\mathrm{Hx}}$ and $\eta_{\mathrm{H}}$, on the moment coefficients, $C_{\mathrm{M}}$ and $C_{\mathrm{M}}^{*}$, are also plotted in Figs. 19 and 20, respectively. In conclusion it can be stated that by decreasing the hydrodynamic force coefficients, $C_{\mathrm{Hx}}$ and $C_{\mathrm{Hy}}$, for constant values of the hydrodynamic efficiency coefficient, the dimensionless VMG increases and the moment coefficient, $C_{\mathrm{M}}^{*}$, decreases;
Fig. 12 Moment coefficient versus the parameter $A$, which represents the value of the maximum camber for $B=0.016$ (solid line) and $B=0.15$ (dashed line). The hydrodynamic coefficient values are $\eta_{\mathrm{H}}=3, C_{\mathrm{Hx}}=0.1$ and $K=100$. The dotted line represents the maximum moment coefficient the yacht can withstand
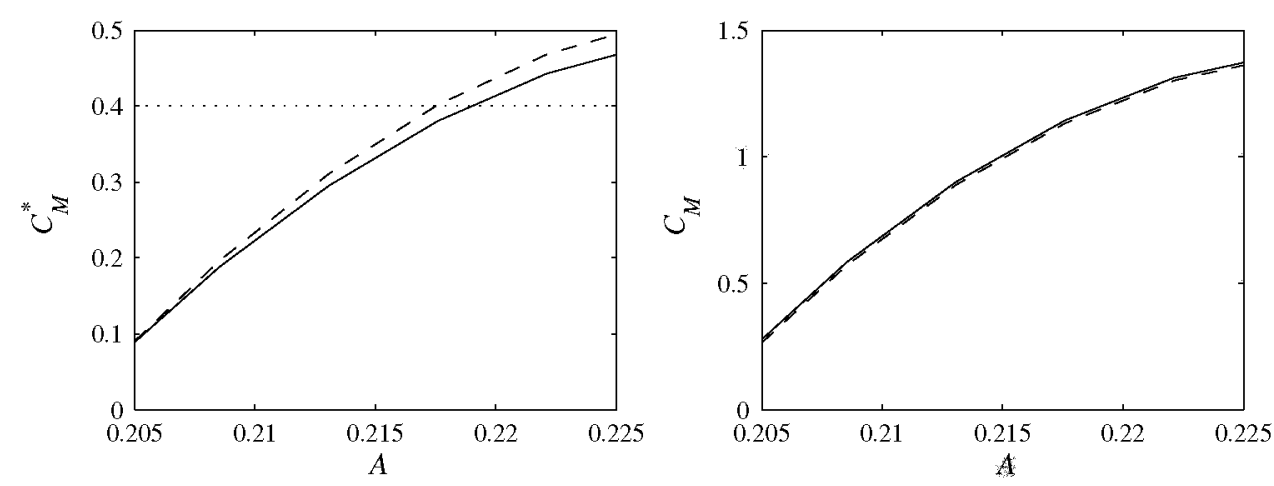
Fig. 13 VMG and aerodynamic coefficients of the sail for the optimal sailing point versus one of the parameters of the polar of the sail, $B$, representing the maximum camber position along the mast direction. The values are obtained for different values of the hydrodynamic force coefficient $\left(C_{\mathrm{Hx}}=0.1\right.$ for the solid line, $C_{\mathrm{Hx}}=0.2$ for the the dotted line), and for $\eta_{\mathrm{H}}=3$, $K=100, A=0.225$ dashed line and $C_{\mathrm{Hx}}=0.3$ for
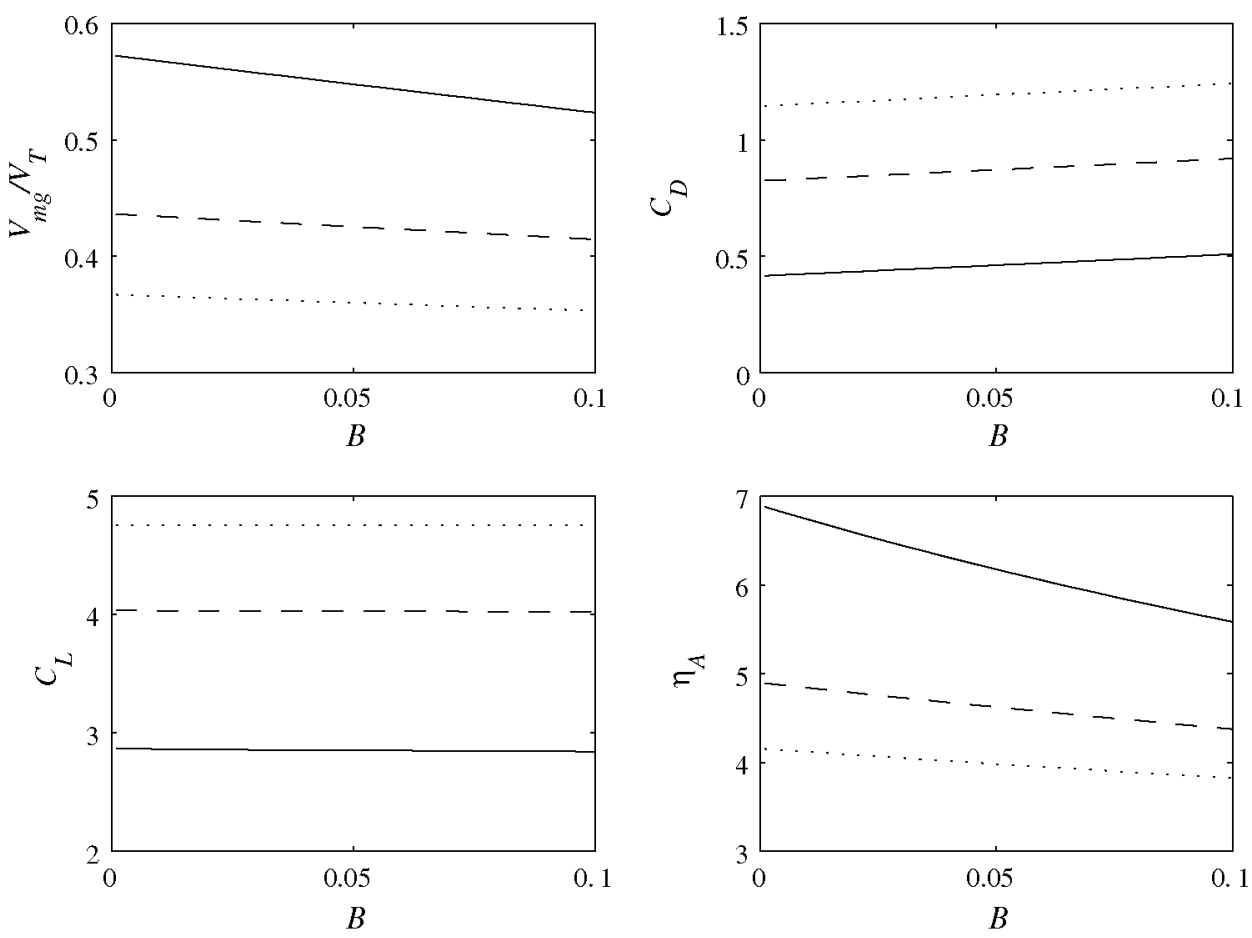

Fig. 14 VMG and aerodynamic coefficients of the sail for the optimal sailing point versus one of the parameters of the polar of the sail, $B$, representing the maximum camber position along the mast direction. The values are obtained for different values of the hydrodynamic efficiency coefficient $\left(\eta_{\mathrm{H}}=2\right.$ for the solid line, $\eta_{\mathrm{H}}=3$ for the dashed line and $\eta_{\mathrm{H}}=4$ for the dotted line), and for $C_{\mathrm{Hx}}=0.1$, $K=100, A=0.225$
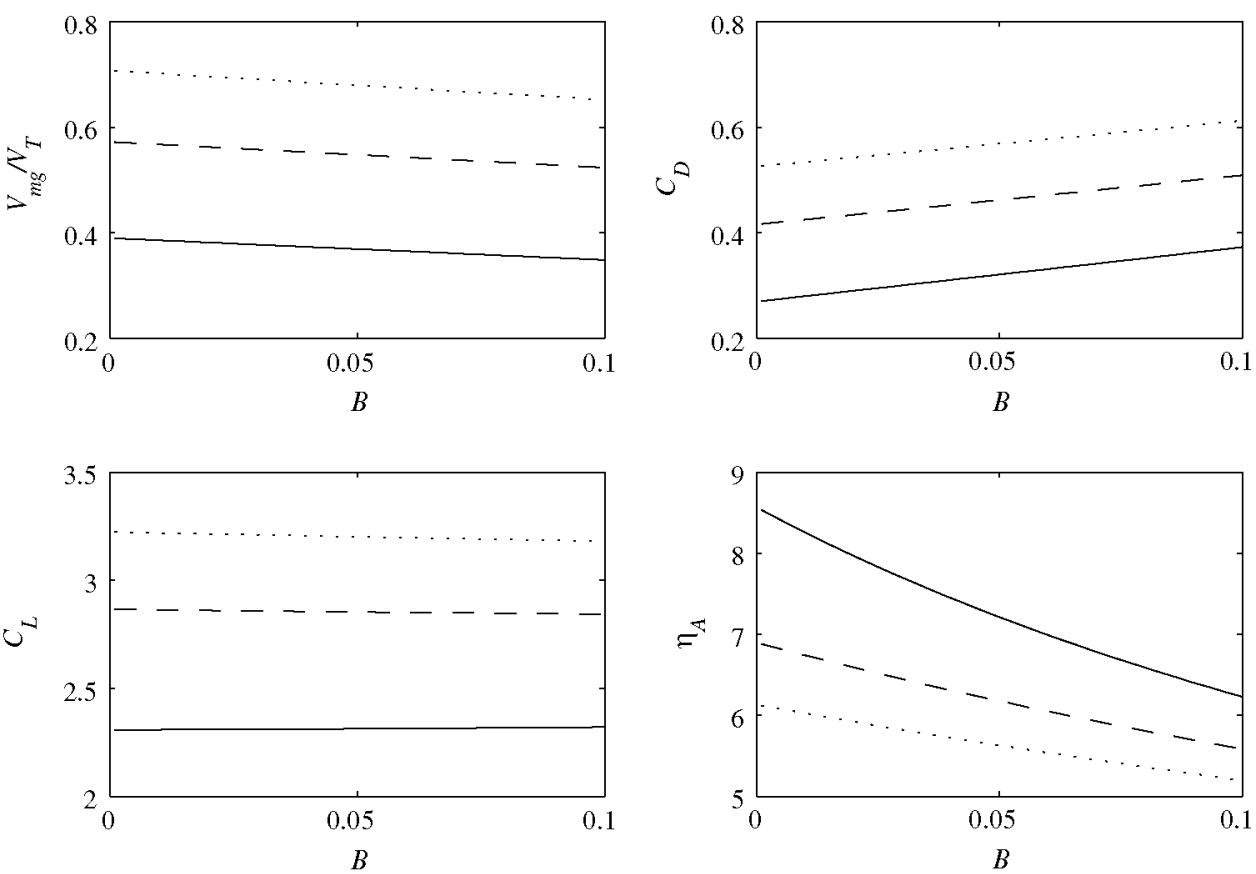

both effects being beneficial for the design. On the other hand, for constant values of the force coefficient, $C_{\mathbf{H x}}$, increasing the hydrodynamic coefficient, $\eta_{\mathrm{H}}$, increases the dimensionless VMG, while the moment coefficient, $C_{\mathrm{M}}^{*}$, decreases for $A>A_{0}$ and increases for $A<A_{0}$. For the case studied $A_{0}=0.213$. It should be pointed out that in this case, in which $C_{\mathrm{Hx}}$ is being held constant, the increase in the hydrodynamic efficiency coefficient can only be done decreasing the hydrodynamic force coefficient, $C_{\mathrm{Hy}}$.

\section{Parametric aerodynamic study of the polar of the sail}

A study on the aerodynamic force and moment coefficients is done using a vortex lattice method [12]. The aerodynamic coefficients are calculated as a function of the angle of attack of the sail. As explained in the previous section a crucial relation for optimizing the VMG is the polar of the sail, provided that the heeling moment does not exceed a maximum value. 
Fig. 15 Moment coefficient versus the parameter $B$, which represents the maximum camber position along the mast direction. The values are obtained for different values of the hydrodynamic force coefficient $\left(C_{\mathrm{Hx}}=0.1\right.$ for the solid line, $C_{\mathrm{Hx}}=0.2$ for the dashed line and $C_{\mathrm{Hx}}=0.3$ for the dotted line), and for $\eta_{\mathrm{H}}=3$, $K=100, A=0.225$
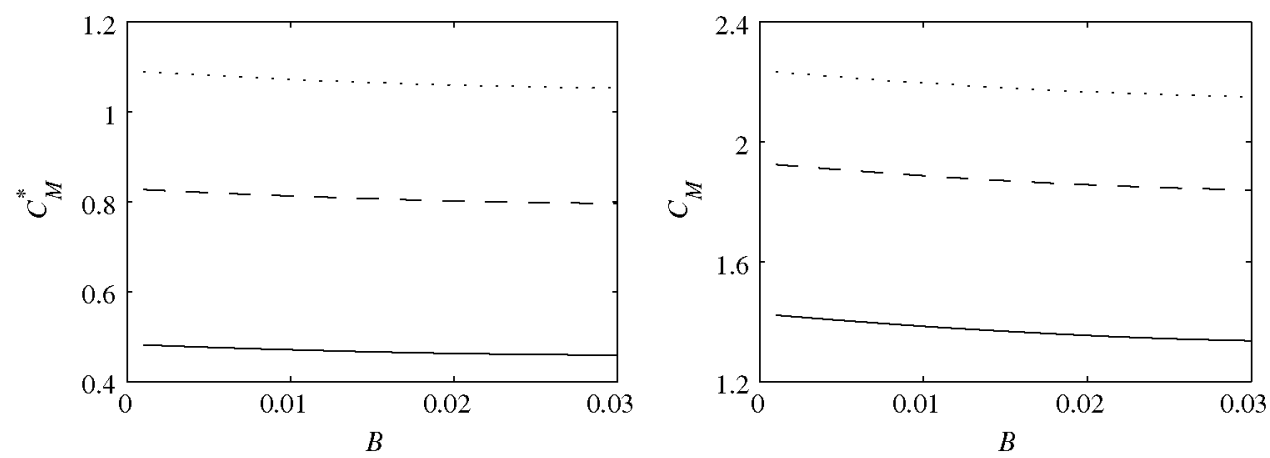

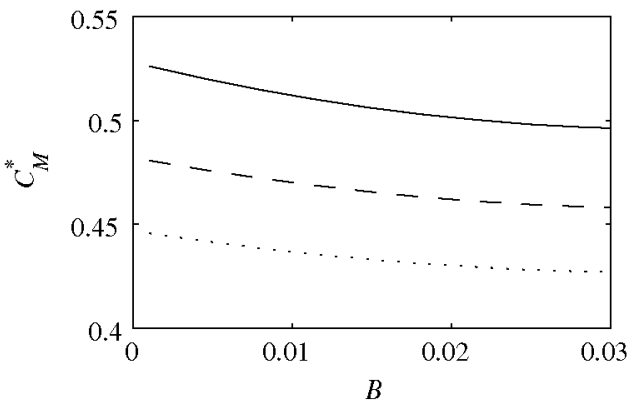

Fig. 16 Moment coefficient versus the parameter $B$, which represents the maximum camber position along the mast direction. The values are obtained for different values of the hydrodynamic efficiency

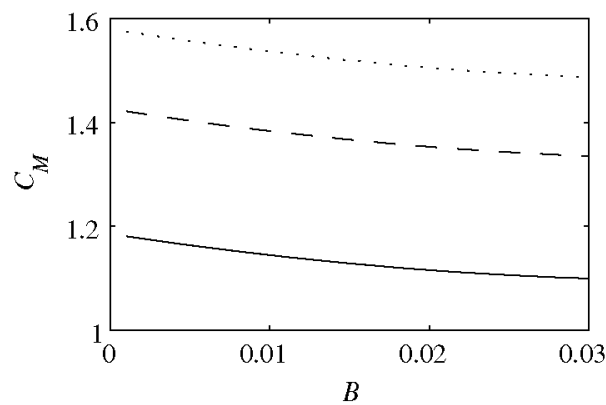

coefficient $\left(\eta_{\mathrm{H}}=2\right.$ for the solid line, $\eta_{\mathrm{H}}=3$ for the dashed line and $\eta_{\mathrm{H}}=4$ for the dotted line), and for $C_{\mathrm{Hx}}=0.1, K=100, A=0.225$
Fig. 17 VMG and aerodynamic coefficients of the sail for the optimal sailing point versus one of the parameters of the polar of the sail, $A$, representing the maximum camber of the sail. The values are obtained for different values of the hydrodynamic force coefficient $\left(C_{\mathrm{Hx}}=0.1\right.$ for the solid line, $C_{\mathrm{Hx}}=0.2$ for the dashed line and $C_{\mathrm{Hx}}=0.3$ for the dotted line), and for $\eta_{\mathrm{H}}=3, K=100$, $B=0.016$
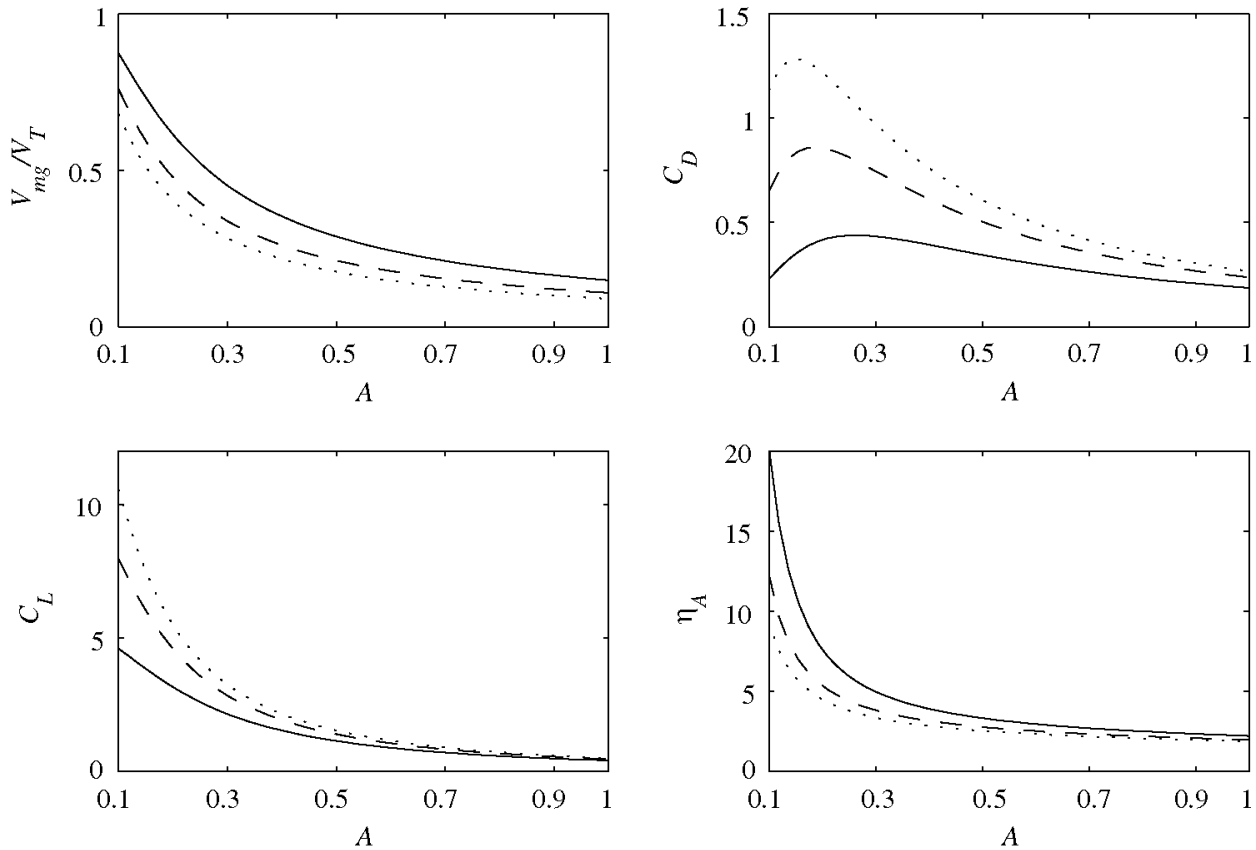

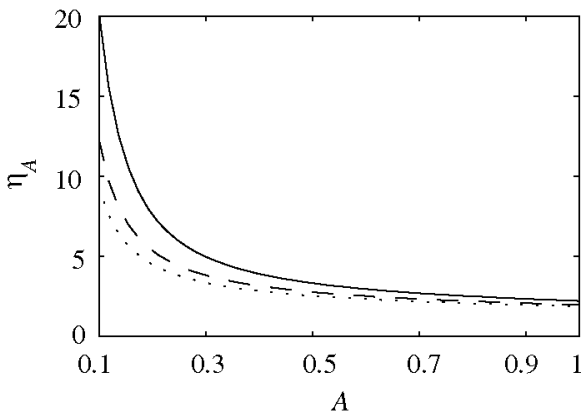

The sail geometry of a Tornado, the Olympic class catamaran, has been obtained from a three-dimensional analysis based on photographs of the craft taken while it was sailing to windward. The geometry has been characterized by a set of parameters, some of which are later modified in order to study their influence on the sail performance. The geometry of the sail studied can be defined by the following parameters: the sail span, $b$; the profile chord, $C$; the chord of the root section of the sail, $C_{\mathrm{r}}$; the chord of the tip section of the sail, $C_{t}$; the maximum camber of the profile expressed as a percentage of its chord length, $m$; the location of the maximum camber along the chord of the profile expressed as a 
Fig. 18 VMG and aerodynamic coefficients of the sail for the optimal sailing point versus one of the parameters of the polar of the sail, $A$, representing the maximum camber of the sail. The values are obtained for different values of the hydrodynamic efficiency coefficient $\left(\eta_{\mathrm{H}}=2\right.$ for the solid line, $\eta_{\mathrm{H}}=3$ for the dashed line and $\eta_{\mathrm{H}}=4$ for the dotted line), and for $C_{\mathrm{Hx}}=0.1, K=100$, $B=0.016$

Fig. 19 Moment coefficient versus the parameter $A$, which represents the maximum camber of the sail. The values are obtained for different values of the hydrodynamic force coefficient $\left(C_{\mathrm{Hx}}=0.1\right.$ for the solid line, $C_{\mathrm{Hx}}=0.2$ for the dashed line and $C_{\mathrm{Hx}}=0.3$ for the dotted line), and for $\eta_{\mathrm{H}}=3$, $K=100, B=0.016$

Fig. 20 Moment coefficient versus the parameter $A$, which represents the maximum camber of the sail. The values are obtained for different values of the hydrodynamic efficiency coefficient $\left(\eta_{\mathrm{H}}=2\right.$ for the solid line, $\eta_{\mathrm{H}}=3$ for the dashed line and $\eta_{\mathrm{H}}=4$ for the dotted line), and for $C_{\mathrm{Hx}}=0.1, K=100$, $B=0.016$
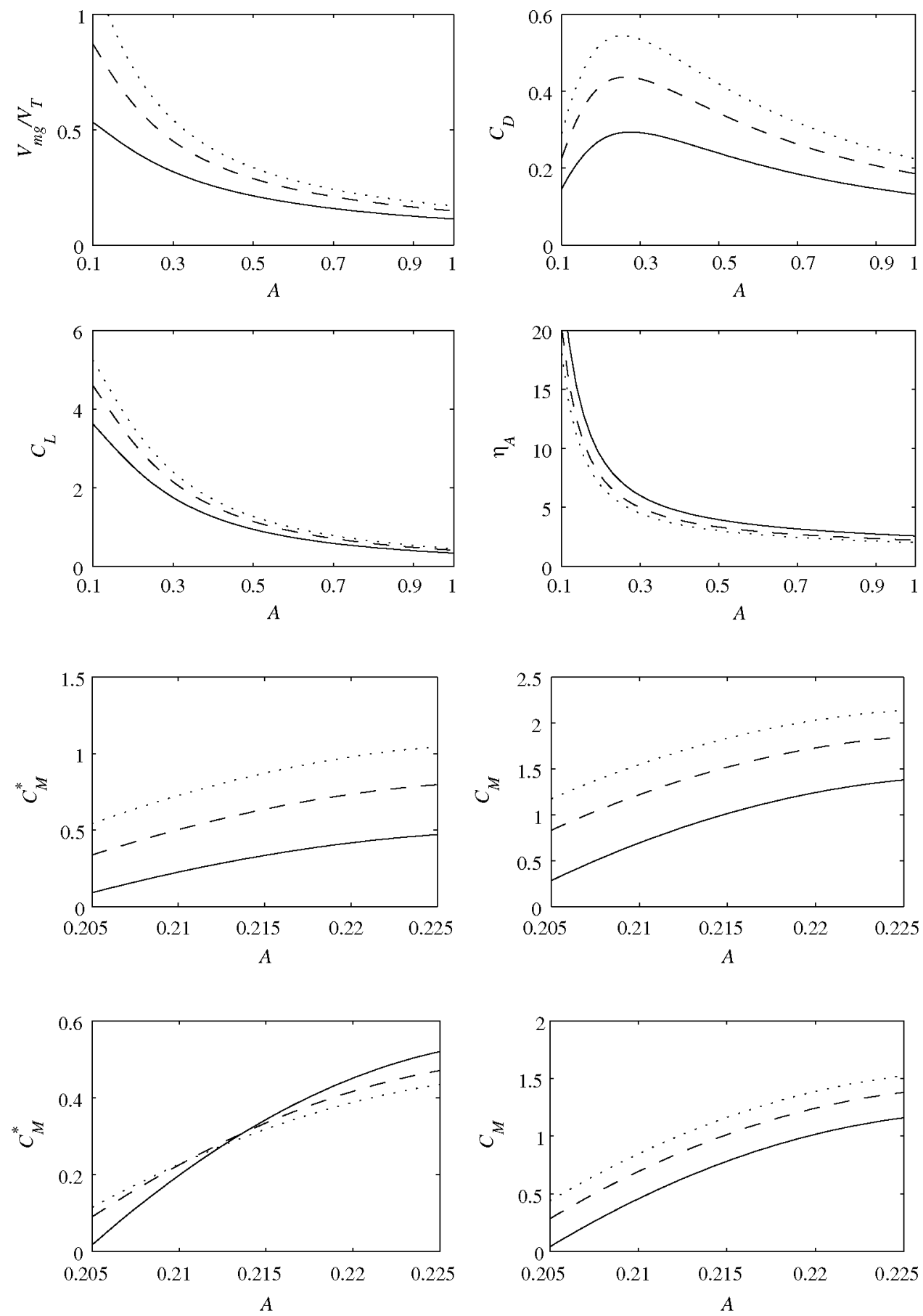

percentage of the chord, $\xi_{\mathrm{m}}$; the location of the profile with maximum camber along the mast direction as a percentage of the sail span, $\eta_{\mathrm{m}}$, the taper ratio defined as $\mu=C_{\mathrm{t}} / C_{\mathrm{r}}$, and the twist angle of each profile, $\tau$.

The data of the sail geometry are given in Fig. 21 where the sail has been divided in ten panels with different geometric characteristics.

The numerical calculations do not simulate the effect of the hull, but they do simulate the effect of the gap between the sail and the deck using a symmetric wing. The size of the mesh used in the numerical calculations was determined with a convergence study in which the lift coefficients calculated with meshes of different sizes were compared. The mesh is determined by the number of sections in the span direction, $N_{y}$, and the number of sections in the chord direction, $N_{x}$. It has to be noticed that the number of elements of the complete mesh is $2 N_{x} N_{y}$, because the sail and its symmetrical have to be considered 


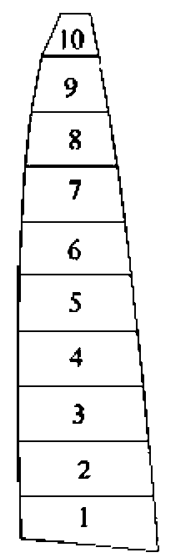

\begin{tabular}{|c|c|c|c|c|c|c|c|}
\hline Panel & Pualition & b) & $\mathbf{r}_{1}$ & $C_{r}$ & $\mu$ & $m$ & $\xi$ \\
\hline & |m| & [m] & 「"1 & $|\mathbf{m}|$ & & $\%$ & $\%$ \\
\hline 1 & $0.00-0.86$ & 0.86 & 3 & $224-218$ & 0.975 & 4 & 50 \\
\hline 2 & 0.86 .1 .73 & 0.86 & 5 & 2.18 .2 .13 & 0.976 & 7 & 40 \\
\hline 3 & 1.73 .2 .59 & 0.86 & 7 & $2.13-2.07$ & 0972 & 9 & 40 \\
\hline 1 & $2.59 \cdot 3.15$ & 0.86 & 10 & $2.07 \cdot 1.98$ & 0958 & 11 & Ad] \\
\hline 5 & $3.45-431$ & 0.86 & 13 & $1.98-1.87$ & 0942 & 11 & A10 \\
\hline 6 & $4.31-5.18$ & 0.86 & 16 & $1.87-1.77$ & 0948 & 10 & 40 \\
\hline 7 & $5.28-6.04$ & 0.86 & 19 & $1.77 \cdot 1.6 \mathrm{I}$ & 0.906 & 9 & 40 \\
\hline 8 & $604-6.90$ & 0.86 & 20 & $1.67 \cdot 1.36$ & 0.847 & 8 & 50 \\
\hline 9 & $6.90-7.76$ & 0.86 & 23 & $1.36-1.03$ & 0.759 & 6 & 40 \\
\hline 10 & $7.76 \cdot 8.43$ & 0.67 & 25 & $1.03-0.54$ & o s2s & 4 & 10 \\
\hline
\end{tabular}

Fig. 21 Geometric characteristics of the ten panels of the sail

in the model. The numerical meshes used were $N_{y}=4,8$, 12 for different values of $N_{x}$; the results obtained are represented in Fig. 22. From these results the dimension of the selected mesh was: $N_{y}=12$ and $N_{x}=16$ which gives good results at reasonable computational cost. The lift coefficient and the drag coefficient calculated are presented in Fig. 23 as a function of the angle of attack of the sail and the polar of the sail is also represented in the figure. It is important to notice that the vortex lattice method does not account for the viscosity of the fluid, and for large angles of attack and whenever the flow is not attached to the sail the experimental results cannot be compared with the numerical results. In Fig. 6 the polar of the sail is plotted along with the curves of constant dimensionless VMG, showing the maximum obtainable value with this sail configuration.

Different sail shapes can be obtained by varying relevant geometric parameters, and the same aerodynamic analysis has been done for the resulting sails in order to obtain the influence of the sail shape on the performance of the yacht.

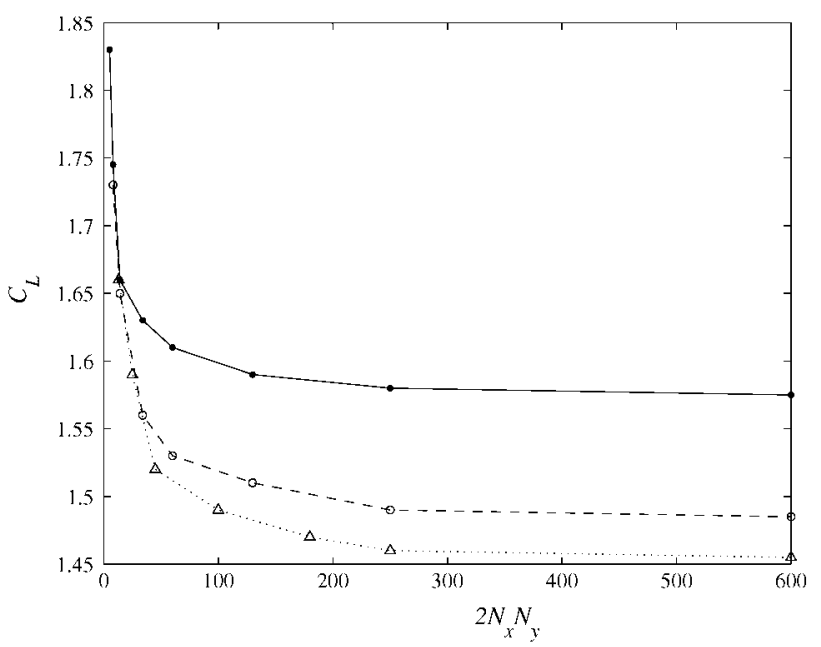

Fig. 22 Lift coefficient for the sail with meshes of different sizes: solid line $N_{y}=4$, dashed line $N_{y}=8$, dotted line $N_{y}=12$
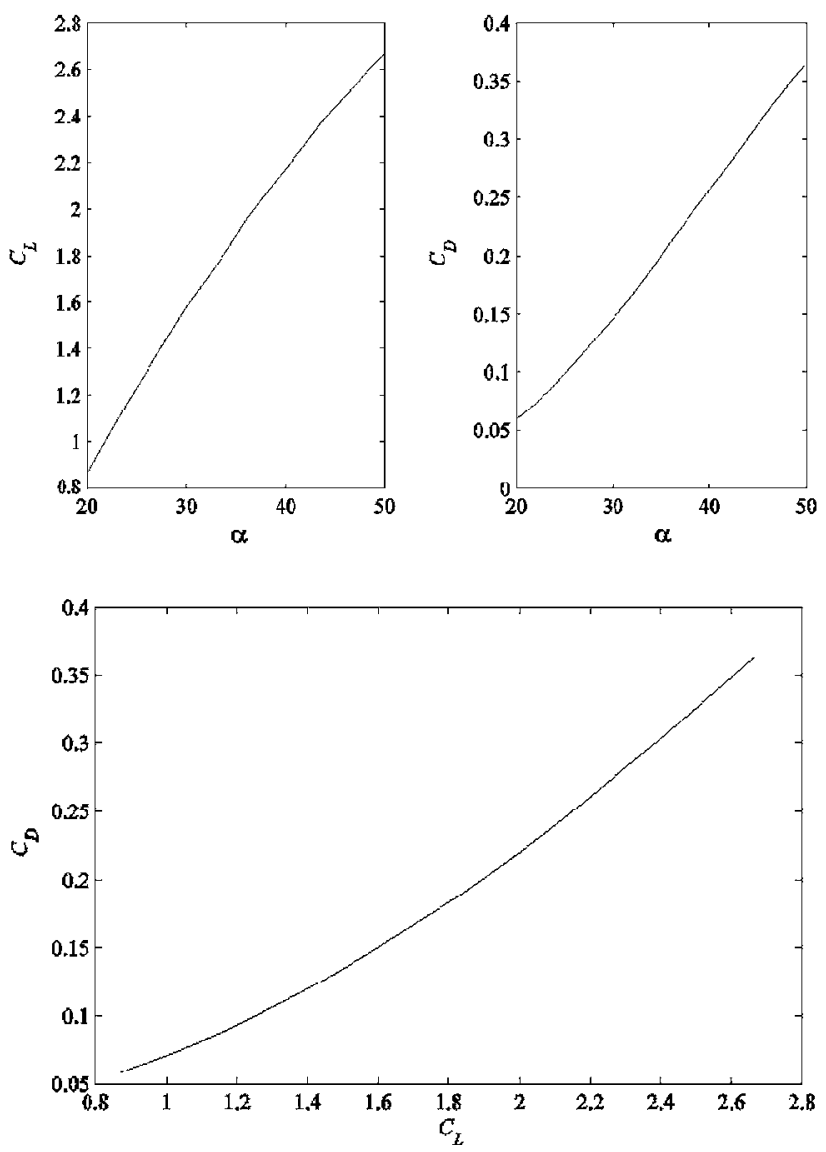

Fig. 23 Lift coefficient $\left(C_{\mathrm{L}}\right)$ and drag coefficient $\left(C_{\mathrm{D}}\right)$ versus the angle of attack $(\alpha)$ and polar of the sail

The geometric characteristics studied here are the twist of the sail and its camber. The latter is studied using three parameters, each measuring the position of the maximum camber along the mast direction, the position of the maximum camber along the chord direction and the value of the maximum sail camber. The sweep angle of the sail was also studied but it did not show a significant influence on the results obtained.

\subsection{Analysis of the influence of the sail twist}

The geometry of the sail has a linear twist law $\tau(z)=m_{\tau} z$, $z$ being measured in meters, $\tau$ in $\mathrm{deg}$ and the slope $m_{\tau}=3^{\circ} / \mathrm{m}$. The twist has been defined positive if the geometric angle of attack of the section profile is smaller than the geometric angle of attack of the root section profile of the sail in order to avoid using negative twist values. Four sails, two of them having bigger values of the twist slope than the nominal sail and two of them having smaller values, $m_{\tau}=0,2,4$ and $6 \% \mathrm{~m}$, have been studied. The grids used to define their geometry are represented in Fig. 24 and their lift and drag coefficients are represented as a function of the sail angle of attack in Fig. 25. The drag 


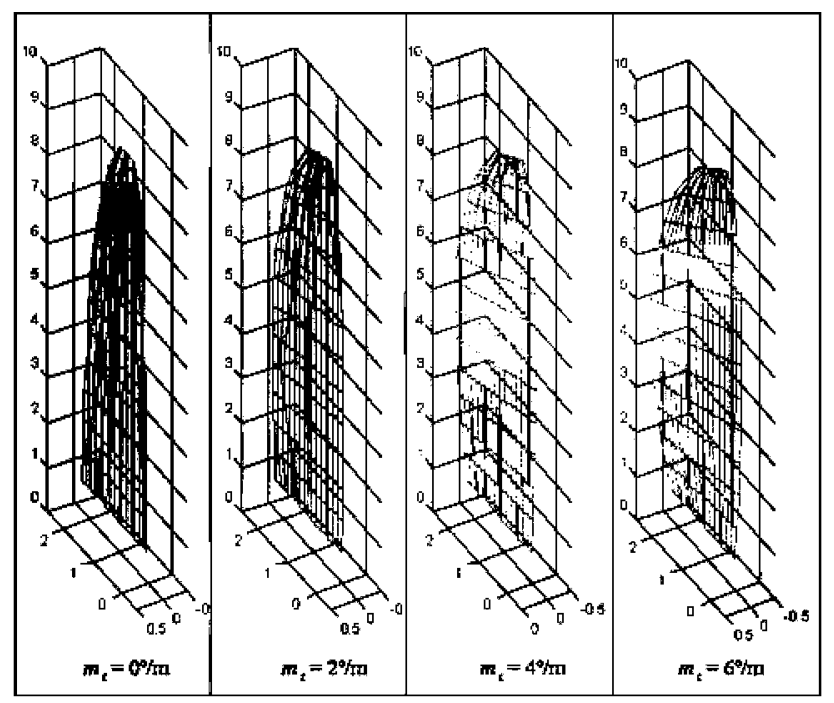

Fig. 24 Grids generated to represent four sails with different twist slopes

coefficient is also represented in Fig. 26 as a function of the lift coefficient along with the curves corresponding to constant values of the heeling moment coefficient. As explained above these curves are useful because the heeling moment is mainly due to the aerodynamic forces and cannot exceed a maximum value. Obviously, the moment coefficient increases for increasing lift coefficients and the same lift coefficient can be obtained with a smaller moment coefficient using bigger twist slopes, at the expense of a higher drag coefficient. From these results it can be deduced that to reach the maximum VMG, for a given $\vec{V}_{\mathrm{T}}$, the minimum twist slope should be used, but the heeling moment may impose a restriction in the optimum value.

\subsection{Analysis of the influence of the sail camber}

In this section, two parameters measuring the sail camber are studied: the position of the maximum camber in the mast direction and the value of the maximum camber.

In order to study the influence of the position of the maximum camber along the mast direction three different sails have been considered, all of them having the maximum camber positioned along the line situated at $\xi_{\mathrm{m}}=40 \%$ from the leading edge of the sail with the maximum camber value $m=35 \%$, as represented in Fig. 27. The values considered for the position of the profile with maximum camber along the mast direction are $\eta_{\mathrm{m}}=10,50$ and $90 \%$.

The value of the maximum camber of the sail selected, $m=35 \%$, is too high for a real sail. A mainsail with that camber would have a large separation region and inviscid results would not be accurate. We have used such a big
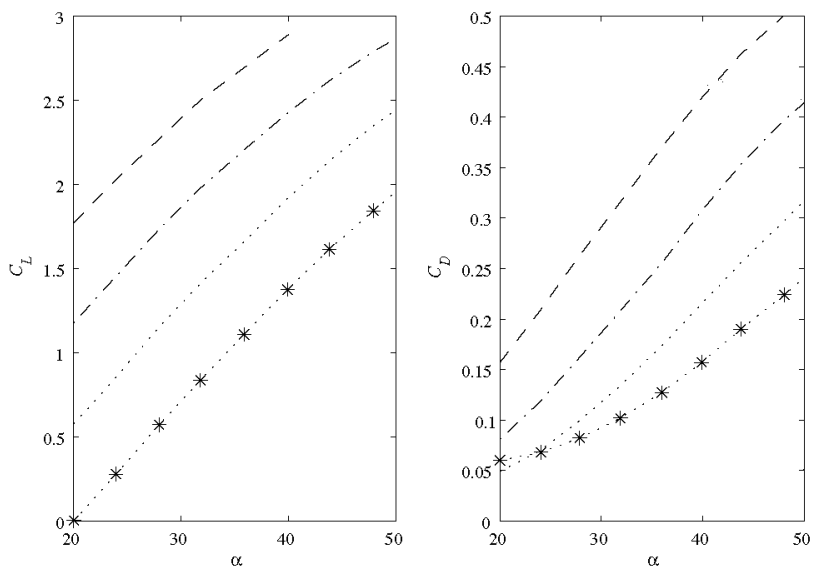

Fig. 25 Lift coefficient $\left(C_{\mathrm{L}}\right)$ and drag coefficient $\left(C_{\mathrm{D}}\right)$ versus the angle of attack of the sail $(\alpha)$. Different line styles have been selected for different values of the twist slope: $m_{\tau}=0$ dashed line, $m_{\tau}=2$ dotted-dashed line, $m_{\tau}=4$ dotted line and $m_{\tau}=6$ dotted with stars

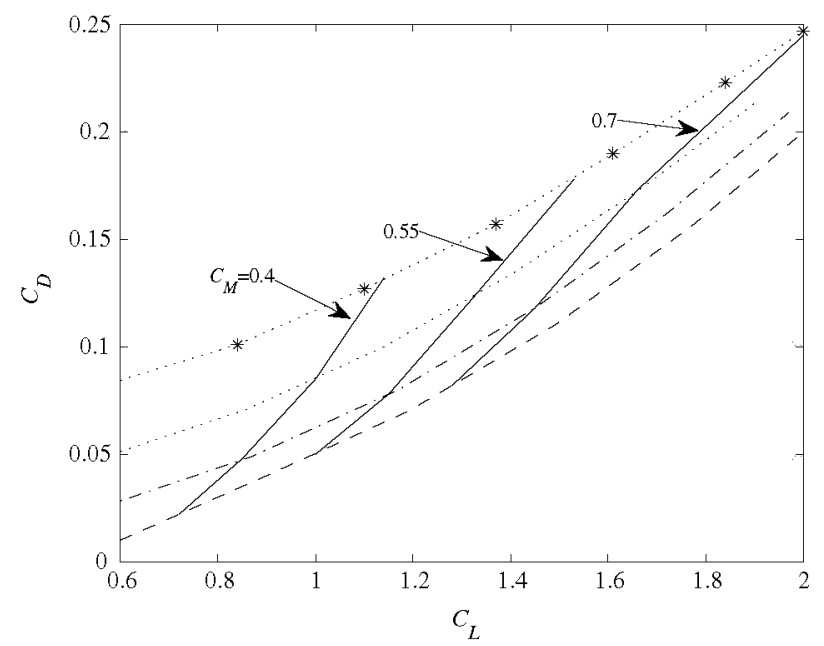

Fig. 26 Lift and drag coefficients of sails with different values of the twist slope. Different line styles have been selected for different values of the twist slope: $m_{\tau}=0$ dashed line, $m_{\tau}=2$ dotted-dashed line, $m_{\tau}=4$ dotted line and $m_{\tau}=6$ dotted with stars. Solid lines represent the configurations providing constant values for the moment coefficient

value only to better illustrate its influence in the results, but the results are only applicable if the value of the sail camber is small enough to avoid having large separation regions in the sail.

Finally, three sails with the same geometry as the one obtained from the sailing configuration but with different maximum camber values are analyzed, $m=2,16$ and $35 \%$.

\subsubsection{Maximum camber position along the mast direction}

The aerodynamic drag coefficient is represented in Fig. 28, for the three sails with $\xi_{\mathrm{m}}=10,50$ and $90 \%$, as a function of the lift coefficient; the curves corresponding to constant 


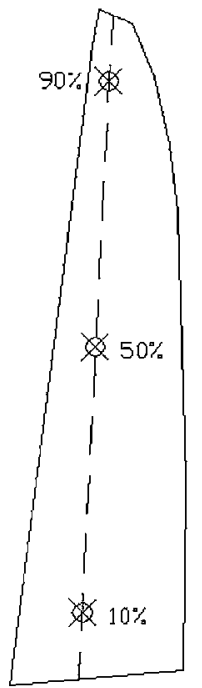

(a)

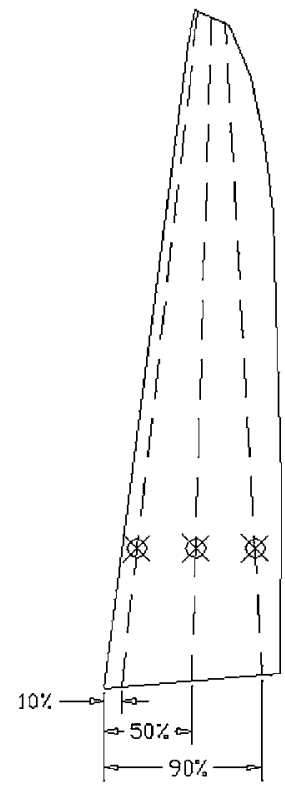

(b)
Fig. 27 Position of the maximum camber of the sail

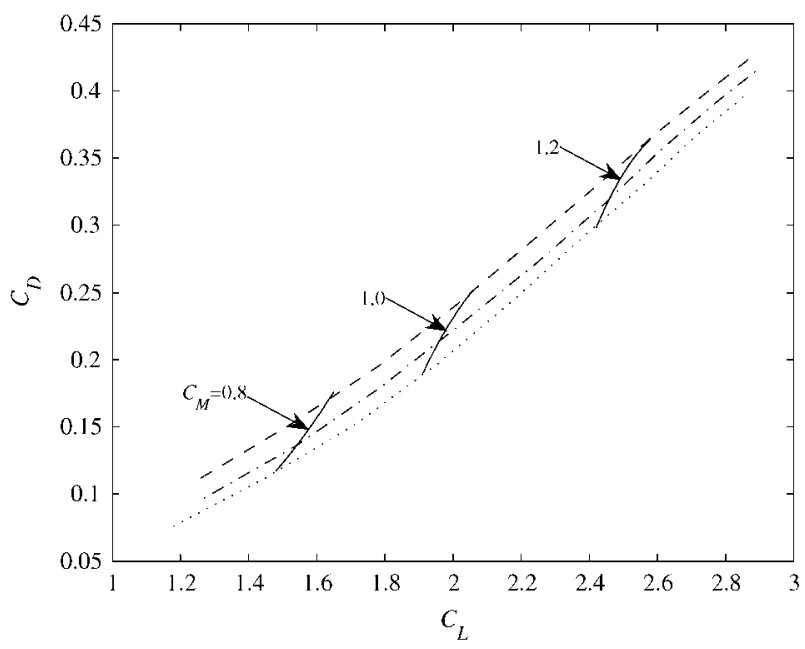

Fig. 28 Drag coefficient versus the lift coefficient for three sails with their maximum camber section situated at different distances from the root section. Different line styles represent different values of the distance of the section with maximum camber from the root section expressed as a percentage of the sail length: $10 \%$ dashed line, $50 \%$ dotted-dashed line and $90 \%$ dotted line. Curves corresponding to constant values of the moment coefficient are represented with solid lines

values of the moment coefficient are also represented in the figure. The influence of the studied parameter is very similar to the influence of the slope twist studied before. As the maximum camber gets farther away from the root of the sail, the curves move in the right direction for maximizing the VMG but at the price of increasing, for a fixed value of the lift coefficient, the moment coefficient which, as

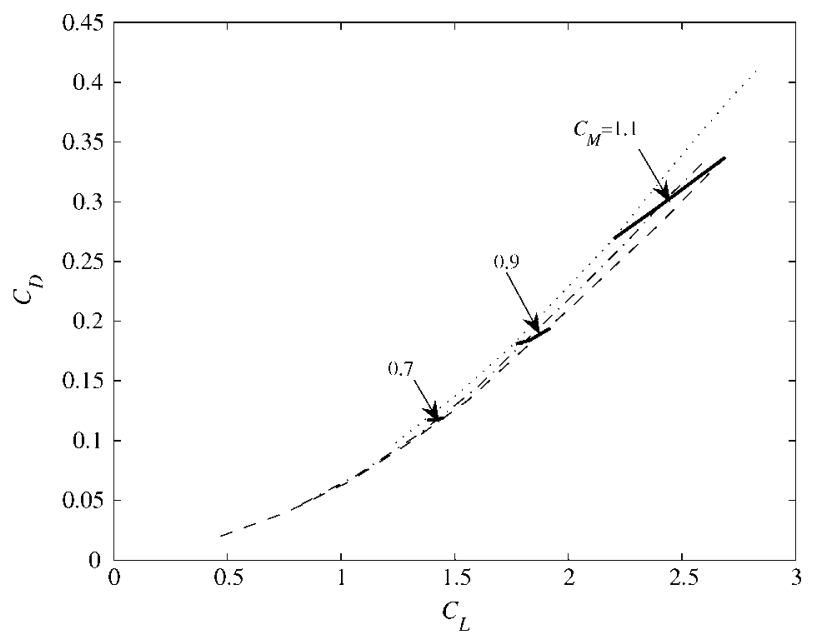

Fig. 29 Drag coefficient versus the lift coefficient for three sails with different values of maximum camber. The curves are represented with different line styles for the different values of the maximum camber expressed as a percentage of the chord: $2 \%$ dashed line, $16 \%$ dashed dotted line and $35 \%$ dotted line. The curves obtained for constant values of the moment coefficient are also represented with solid lines

already mentioned, cannot be freely increased because the boat would capsize. If the polar of the sail is fitted using a parabola as explained in Sect. $2, C_{\mathrm{D}}=B+A^{2} C_{\mathrm{L}}^{2}$, the effect of moving the maximum camber towards the tip of the sail does not change $A$ but decreases $B$.

\subsubsection{Maximum camber value}

In the same way as above, three sails with different values of their maximum camber are analyzed and their drag coefficients are represented in Fig. 29 as a function of their lift coefficients. It can be concluded from the figure that flattening the sail leads to better performance of the yacht in terms of increasing the VMG. The curves obtained for constant values of the moment coefficient are also represented in the figure, and it can be observed how this coefficient decreases as the sail is flattened, which is also beneficial in terms of maximizing the VMG. If the polar of the sail is fitted using a parabola as explained in Sect. 2, $C_{\mathrm{D}}=B+A^{2} C_{\mathrm{L}}^{2}$, the effect of increasing the maximum camber of the sail does not change $B$ but increases $A$.

\section{Experimental aerodynamic study of the aerodynamic coefficients of the sail}

An experiment campaign was performed to contrast the numerical results, which do not account for the viscosity effects. Viscosity has two main effects: an increase in the drag coefficient due to friction and to stream separation, and a decrease in the lift coefficient due to stream separation. The drag increase due to friction can be easily 

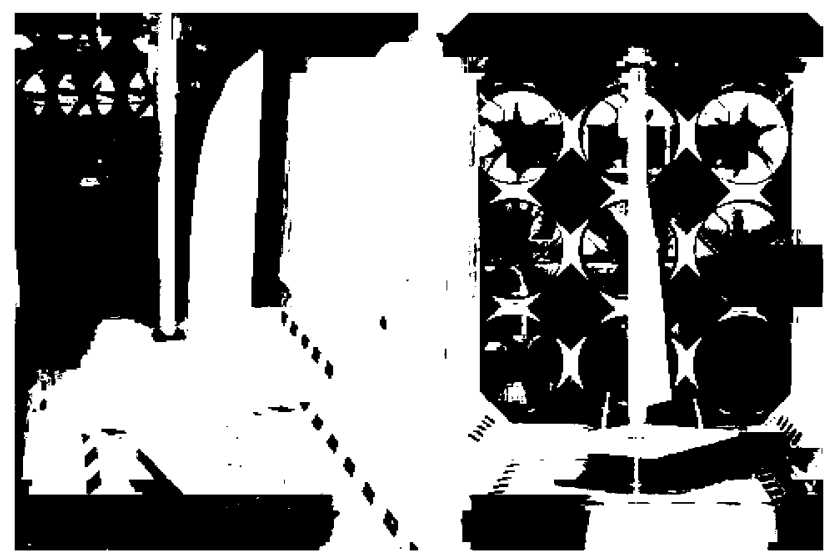

Fig. 30 Sail mounted in the A9 Wind Tunnel

accounted for using $C_{\mathrm{D}}=C_{\mathrm{D} 0}+C_{\mathrm{Di}}$ where $C_{\mathrm{D} 0}$ is the frictional drag coefficient, and $C_{\mathrm{Di}}$ the induced drag coefficient calculated with the numerical method. The drag increase due to stream separation depends on the angle of attack, and therefore on the lift coefficient. But as the flow is detached in the intrados of the sail for all the angles of attack, due to the mast-sail geometry, the increase can also be approximated with the same coefficient. $C_{\mathrm{D} 0}$, which is, therefore, approximated from the experimental data as the drag coefficient for zero lift $C_{\mathrm{D} 0}=C_{\mathrm{D}}\left(C_{\mathrm{L}}=0\right)$. Although other effects are not easily accounted for it will be demonstrated that the numerical results correctly predict the trends in the polars with the different parameters.

In order to perform wind tunnel experiments the rig geometry was reproduced in 1:7 scale. The mast core was manufactured from commercial threaded rod and a bronze tube, which was conformed so as to reproduce the profile of the original mast, covers it. The battens supporting the sail are ten rods of steel with different lengths and cambers. The sail was made of plywood wood $1.2 \mathrm{~mm}$ thick, and was tied to the battens reproducing the desired camber and twist. Three sets of battens were made, each set corresponding to a sail with different camber properties. The mast of the sail was fixed to a wooden base, and four shrouds and two stays assure the rigidity needed. The gap between the sail and the hull was reproduced in the model. The lower part of the model was an iron work which fixes the set to a rotating platform and to the load cell whereupon the measurements were made.

Measurements were carried out in the A9 Wind Tunnel at the E.T.S.I. Aeronáuticos, the pictures of the sail in the wind tunnel as shown in Fig. 30. The tunnel test chamber is $1.5 \mathrm{~m}$ in width and $1.8 \mathrm{~m}$ in height. The testing model was anchored to a NEWPORT RV120-PP-HL rotating platform, which was mounted on a six-component straingauge balance, as sketched in Fig. 31.

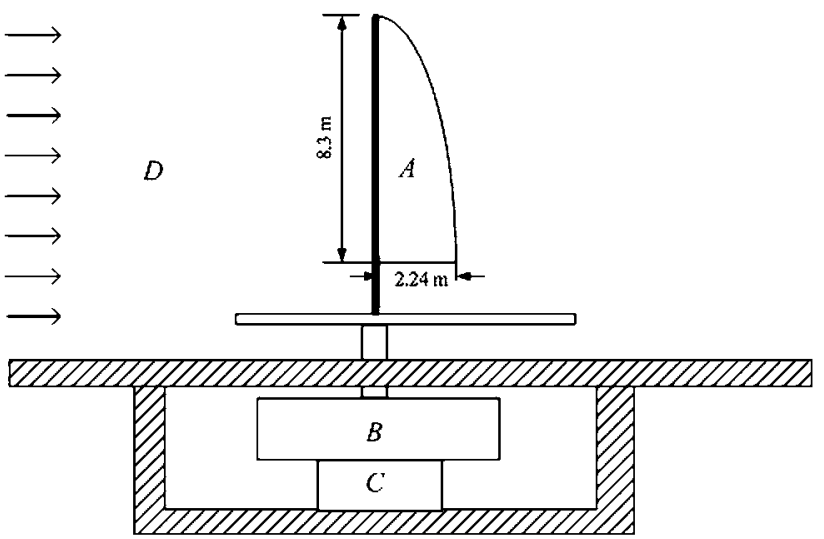

Fig. 31 Sketch of the experimental arrangement: a sail model, b rotating platform, $\mathbf{c}$ strain-gauge balance and $\mathbf{d}$ wind tunnel test chamber

The rotating platform allows the control of the model's angle of attack with accuracy better than $\pm 1^{\circ}$. The rotating platform and the balance were placed inside a tight chamber located under the wind-tunnel floor.

The dynamic pressure inside the test chamber was measured by a calibrated air flow 048 Pitot tube connected to a Schaewitz Lucas P3061-2 WG pressure transducer.

It must be pointed out that even in the worst case (the rig at a $90^{\circ}$ of attack), the frontal area of the model is smaller than $5 \%$ of the wind tunnel cross section, so that no provisions for blockage correction of the measured results were considered. Experiments were performed in a lowturbulence, uniform flow (the turbulence intensity being around $2.5 \%$ ).

Each sail model was tested in the wind tunnel measuring the forces and the moments generated on it. For each test run, the angle of attack, $\alpha$, between the sail boom and the wind direction was varied from $5^{\circ}$ to $40^{\circ}$ in $2.5^{\circ}$ increments. The forces and moment coefficients were determined from the measured forces. Three tests were made varying the twist and other three varying the maximum camber of the sail.

\subsection{Influence of the sail twist}

The results of the three tests in which the twist slope of the sail, $m_{\tau}$, was varied are presented in Figs. 32 and 33. In Fig. 32 the experimental results are represented along with the numerical results modified to account for the nonpotential drag coefficient due to flow separation. The numerical results have been modified including the appropriate value of $B$, which is obtained from the experimental results. The experimental and the modified numerical curves show a good correlation up to the stall, $C_{\mathrm{Lmax}} \cong 1.2-1.6$. 


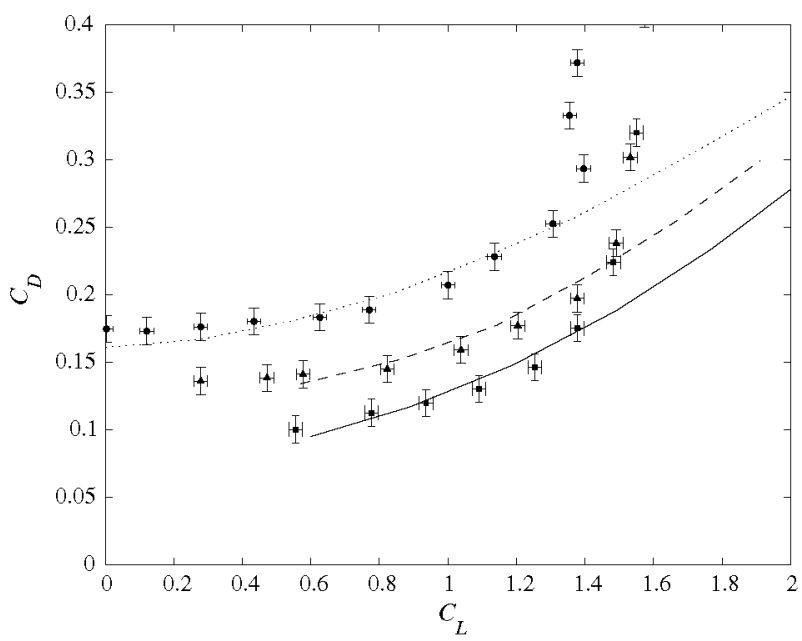

Fig. 32 Drag coefficient versus the lift coefficient for three sails with different values of the twist slope. The experimental results are represented with different marker styles corresponding to different values of the twist slope with the following code: $0 \% \mathrm{~m}$ squares, $3 \% \mathrm{~m}$ triangles and $6 \% \mathrm{~m}$ circles. The lines correspond to the numerical data: $0 \% \mathrm{~m}$ solid line, $3 \%$ dashed line and $6 \% \mathrm{~m}$ dotted line

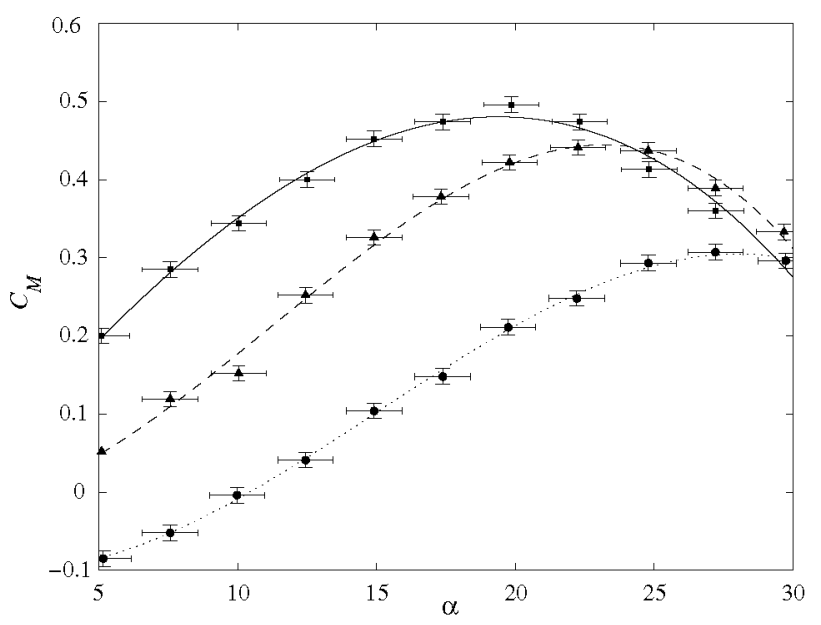

Fig. 33 Aerodynamic moment coefficient versus the angle of attack of the sail for three sails with different values of the twist slope. The experimental results are represented with different marker styles corresponding to different values of the the twist slope with the following code: $0 \% \mathrm{~m}$ squares, $3 \% \mathrm{~m}$ triangles and $6 \% \mathrm{~m}$ circles. The lines are curve fits to the experimental results. The different lines styles correspond to the values of the twist slope: $0 \% \mathrm{~m}$ solid line, $3 \%$ dashed line and $6 \%$ dotted line

The variation of the moment coefficient, $C_{\mathrm{M}}$, as function of the angle of attack, $\alpha$ is represented in Fig. 33.

\subsection{Influence of the maximum camber}

The results of the tests in which the maximum camber of the sail has been varied are represented in Figs. 34 and 35.

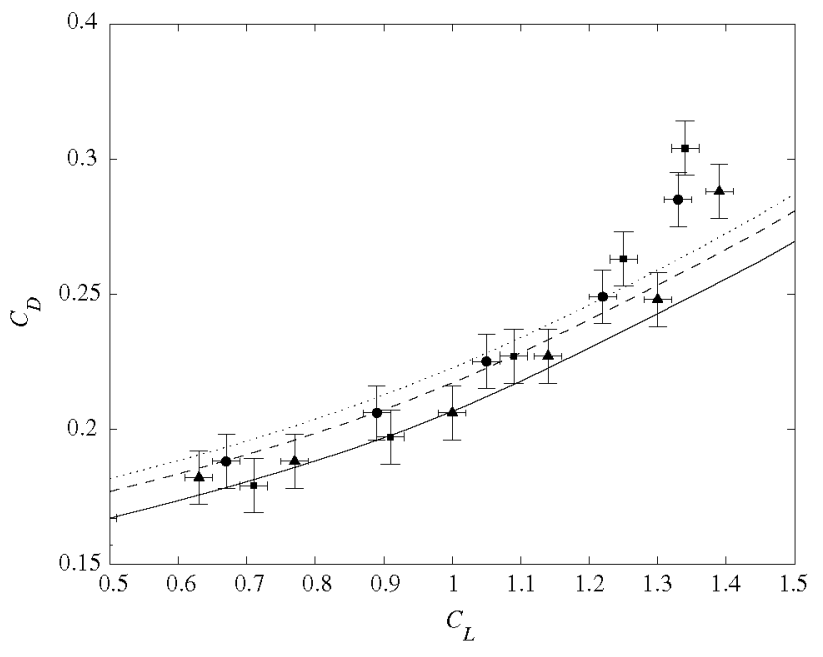

Fig. 34 Drag coefficient versus the lift coefficient for three sails with different values of maximum camber. The experimental results are represented with different marker styles corresponding to different values of the maximum camber of the sail, expressed as a percentage of the chord length with the following code: $0 \%$ squares, $5 \%$ triangles and $10 \%$ circles. The lines correspond to the numerical data: $0 \%$ solid line, $5 \%$ dashed line and $10 \%$ dotted line

In Fig. 34 the modified numerical results have been included. The numerical results have been modified including the appropriate value of $B$, which is obtained from the experimental results. The experimental and the modified numerical curves show a good correlation up to the stall, $C_{\text {Lmax }} \cong 1.2-1.4$.

The variation of the moment coefficient, $C_{\mathrm{M}}$, as function of the angle of attack, $\alpha$ is represented in Fig. 35. The curves represented verify that the behaviour of the moment coefficient with the variation of the camber is, conceptually, well predicted by the numerical model. It should be pointed out that the curve corresponding to a flat sail changes its behaviour for very small angles of attack, due to the early detachment of the flow.

\section{Conclusions}

The VMG is the variable which has to be maximized when sailing upwind. In order to achieve the optimization of that parameter a relationship between the aerodynamic force coefficients and VMG has been obtained for a given hull. The shape of the curves corresponding to constant values of VMG show that the optimum point for a given sail is the point at which the curve $C_{\mathrm{D}}=f\left(C_{\mathrm{L}}\right)$ is tangent to one of the curves $\mathrm{VMG}=g\left(C_{\mathrm{L}}, C_{\mathrm{D}}\right)=$ constant. Furthermore, the optimum sail shall be the one having a curve $C_{\mathrm{D}}=f$ $\left(C_{\mathrm{L}}\right)$ such that the tangency point with the $\mathrm{VMG}=g\left(C_{\mathrm{L}}\right.$, $C_{\mathrm{D}}$ ) family of curves is obtained for greater values of VMG. 


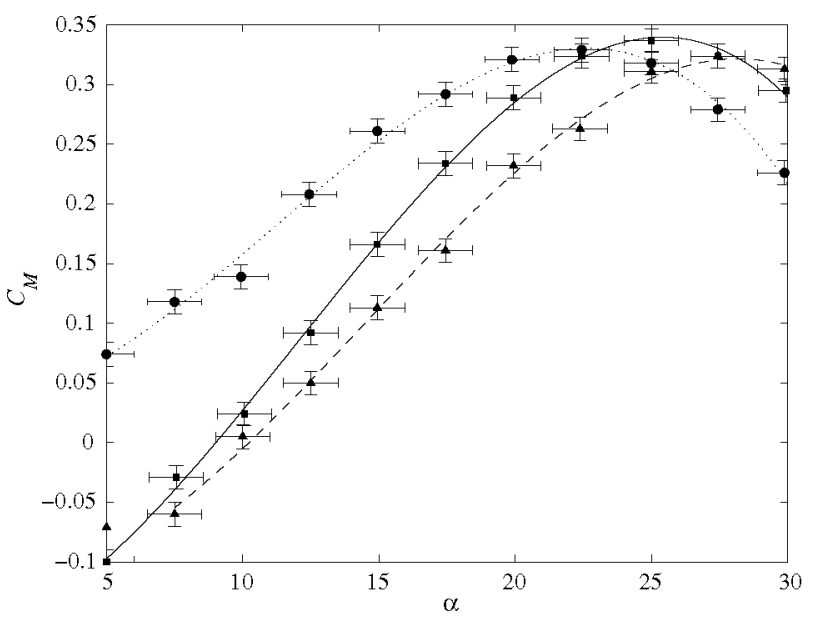

Fig. 35 Aerodynamic moment coefficient versus the angle of attack of the sail for three sails with different values of the maximum camber The experimental results are represented with different marker styles corresponding to different values of the maximum camber of the sail expressed as a percentage of the chord length with the following code: $0 \%$ squares, $5 \%$ triangles and $10 \%$ circles. The lines are curve fits to the experimental results. The different lines styles correspond to the values of the maximum camber: $0 \%$ solid line, $3 \%$ dashed line $6 \%$ dotted line

A parametric study of the aerodynamic coefficients has been calculated for different sails, $C_{\mathrm{D}}=f\left(C_{L} ; m_{\tau}, \xi_{\mathrm{m}}, \eta_{\mathrm{m}}\right.$, $m$ ) with $m_{\tau}$ being the slope of the twist profile, $\eta_{\mathrm{m}}$ the position of the maximum camber along the mast, $\xi_{\mathrm{m}}$ the position of the maximum camber of the sail along the chord and $m$ the value of the maximum camber of the sail. The results obtained are:

1. The twist strongly affects the sail properties. The VMG is maximized with minimum twist. There is an important restriction, however, to the values of this parameter because decreasing it also increases the heeling moment.

2. The position of the maximum camber of the sail along the mast, $\eta_{\mathrm{m}}$, does not affect significantly the shape of the $C_{\mathrm{D}}=f\left(C_{\mathrm{L}}\right)$ curve, and affects the aerodynamic moment coefficient increasing it for increasing values of VMG. The VMG is optimized by increasing the value of $\eta_{\mathrm{m}}$; the influence of this parameter is very similar to the influence of the slope twist, as would be expected. The tendency of the aerodynamic moment coefficient, however, restricts the use of this parameter for increasing the VMG.

3. Decreasing the maximum camber of the sail, $m$, increases the VMG obtainable and decreases the value of the aerodynamic moment coefficient. This parameter can therefore be used to optimize the VMG without introducing heeling moment restrictions.

4. Any change in the sail geometry involving a decrease of the polar parameters $A$ or $B$ leads to an increase of VMG. In the first case the heeling moment coefficient, $C_{\mathrm{M}}^{*}$, also decreases, but in the last case it increases implying a limit to $B$ 's value.

All the conclusions here obtained are qualitative and have been obtained for a given hull configuration.

Acknowledgments This work has been sponsored by the Spanish Consejo Superior de Deportes (CSD) and by the Universidad Politécnica de Madrid (UPM) and is part of a more general endeavour for the study of sails design.

\section{References}

Jackson PS (1985) The analysis of the three-dimensional sails. In: Proceeding of the 10th Canadian congress of applied mechanics Malpede S, Vezza M (2000) Developments of an interactive sail design method. Acta Polytech 4:66-74

Sugimoto T (1996) Theory for inextensible and high aspect-ratio sails. J Wind Eng Ind Aerodyn 63:61-75

Couser P (1998) Computational methods for investigating sail forces-a case study. Yacht Vision '98, Auckland, New Zealand, 29th January-1st February

Locke NJ, Jackson PS (1996) Lift and drag distributions of yacht sails using wake surveys. J Fluid Eng 118:346-351

Sparenberg JA, Wiersma AK (1976) On the maximum thrust of sails by sailing close to wind. J Ship Res 20:98-106

Flay RGJ, Locke NJ, Mallinson GD (1996) Model test of twisted flow wind tunnel designs for testing yacht sails. J Wind Eng Ind Aerodyn 63:155-169

Flay RGJ (1996) A twisted flow wind tunnel for testing yacht sails. J Wind Eng Ind Aerodyn 63:171-182

Marchaj CA (1979) Aero-hydrodynamics of sailing. Adlard Coles, London

Wood CJ, Tan SH (1978) Towards an optimum yacht sail. J Fluid Mech 85:459-477

Jackson PS (1996) Modelling the aerodynamics of upwind sails. J Wind Eng Ind Aerodyn 63:17-34

Margason RJ, Lamar JE, Vortex-lattice FORTRAN Program for estimating subsonic aerodynamic characteristics of complex planforms, NASA TN D-6142, February, 1971 